\title{
Search in Dynamic Scenes: Cueing by Body Parts Leads to Transient Covert Attention and Microsaccade Bias while Whole Silhouettes to Sustained
}

\author{
Nicole X. Han \\ Department of Psychological and Brain Sciences \\ University of California, Santa Barbara \\ xhan01@ucsb.edu
}

\author{
Miguel P. Eckstein \\ Department of Psychological and Brain Sciences \\ Institute for Collaborative Biotechnologies \\ University of California, Santa Barbara \\ miguel.eckstein@psych.ucsb.edu
}




\begin{abstract}
Gaze direction is an evolutionarily important mechanism in daily social interactions. It reflects a person's internal cognitive state, spatial locus of interest, and predicts future actions. Studies have used static head images presented foveally and simple synthetic tasks to find that gaze orients attention facilitates target detection at the cued location in a sustained manner. Little is known about how people's natural gaze behavior, including eyes, head, and body movements, jointly orient covert attention, microsaccades, and facilitate performance in more ecological dynamic scenes. Participants completed a target person detection task with videos of real scenes. The videos showed people looking toward (valid cue) or away from a target (invalid cue) location. We digitally manipulated the individuals in the videos directing gaze to create three conditions: intact (head+body movements), floating heads (only head movements), and headless bodies (only body movements). We assessed their impact on participants' behavioral performance and microsaccades during the task. We show that, in isolation, an individual's head or body orienting toward the target-person direction led to facilitation in detection that is transient in time (200 ms). In contrast, only whole silhouettes led to sustained facilitation (500 ms). Furthermore, observers executed microsaccades more frequently towards the cued direction for valid trials, but this bias was sustained in time only when full silhouettes were present. Together, the results differ from previous findings with foveally presented static heads. In more real-world scenarios and tasks, sustained attention requires the presence of the whole silhouettes of the individuals dynamically directing their gaze.
\end{abstract}




\section{Introduction}

Orienting attention to prioritize the most relevant scene regions is essential to find an object or person in a cluttered visual environment. Studies with simple arrows or boxes predicting a target's location have led to advances in the understanding of attention's performance benefits (Carrasco, 2006, 2011; M. P. Eckstein et al., 2013; Luck et al., 1994; Posner, 1980), mediating computational models (Carrasco, 2006; Dosher \& Lu, 2000; M. P. Eckstein et al., 2002, 2004) and neural mechanisms (Carrasco, 2006; Corbetta \& Shulman, 2002; Gandhi et al., 1999; Garcia et al., 2013; Giesbrecht et al., 2003; Pestilli \& Carrasco, 2005).

In everyday visual search, humans optimize their performance by orienting their attention, not towards arrows and boxes, but towards scene properties and objects that might indicate the probable location of a sought object (Castelhano \& Heaven, 2011; M. Eckstein, 2017; M. P. Eckstein et al., 2006; Koehler \& Eckstein, 2017; Malcolm \& Henderson, 2010; Võ et al., 2019). A person's gaze is an important cue in real-world scenes indicating possible future actions and points of interest (Emery, 2000; Kleinke, 1986). Humans, as early as ten months old (Brooks \& Meltzoff, 2005), and other animals (Bräuer et al., 2005; Bugnyar et al., 2004) use gaze direction to orient attention. Humans are good at perceiving others' gaze directions in daily social interactions (Hessels, 2020). Experiments with gaze cues show signatures of attentional shifts and performance facilitation even when the cue is non-predictive. This involuntary orienting of attention is known as exogenous attention (Egeth \& Yantis, 1997; Jonides \& JONIDES, 1981; Mulckhuyse \& Theeuwes, 2010) in contrast to voluntary endogenous attention triggered by central arrows (Cheal \& Lyon, 1991; Müller \& Rabbitt, 1989; Posner \& Cohen, 1984). However, gaze cues also show signatures of sustained attention, with the facilitation appearing as early as $100 \mathrm{~ms}$ and lasting up to 300-500 ms after the gaze cue onset and then decaying afterward. The sustained effect of gaze cues lasts longer than a peripherally-presented exogenous synthetic cue for which facilitation disappears at 300 ms (Driver et al., 1999; Friesen et al., 2004; McKee et al., 2007; Posner \& Cohen, 1984; Ristic et al., 2007; Theeuwes, 1991). 
One limitation of most previous experiments is that they have not captured the dynamics of the various body cues (gaze, head, body) during natural gazing behavior. The studies have used either static images of faces with various gaze and or body directions (Azarian et al., 2017; Bayliss et al., 2004; Driver et al., 1999; Friesen \& Kingstone, 1998), highly simplified dynamic figures such as moving point-lights (Shi et al., 2010; Wang et al., 2014), videos of a single individual's face or animations (Hermens \& Walker, 2012; Kuhn \& Tipples, 2011). In addition, the gaze cue effects have been typically evaluated with detection tasks of simple stimuli such as letters, dots, and asterisks (Azarian et al., 2017; Driver et al., 1999; Hietanen, 1999; Kingstone et al., 2000) rather than more ecologically valid tasks using real scenes.

More realistic scenarios involve individuals directing gaze that appear away from an observer's fovea and various distances from the observer, making it difficult to infer gaze direction from the visual periphery (Loomis et al., 2008; Palanica \& Itier, 2017). These realistic scenarios involve the integration of multiple dynamic cues: gaze (Azarian et al., 2017; Bayliss et al., 2004; Frischen et al., 2007; Hietanen, 1999, 2002), head orientation (Hessels, 2020; Langton et al., 2004; Mareschal et al., 2013; Otsuka, 2014) and body postures (Azarian et al., 2017; Shi et al., 2010; Wang et al., 2014; Zhao et al., 2014).

Our goal was to investigate how people's natural gaze behaviors contribute to the orienting of attention in a complex, ecologically relevant search task with videos of natural dynamic scenes. To our knowledge, this is the first study that embeds the task in dynamic videos of the real world and explores gaze orienting individuals at various retinal eccentricities and viewing distances.

We focused on viewing angles of faces subtended by people located in the real 3D world at mid to long-range distances (5-30 meters) from the observer. We aim to understand how the individual head, body motion, and joint presence contribute to orienting attention, influence performance, and understand the temporal dynamics of attention for each bodily cue. We used video clips recorded while we instructed multiple actors to look to a specified location in the 
scene that would subsequently present a target person (50\% cue validity) among distractor individuals in different complex dynamic scenes. We created different conditions by erasing heads or bodies of only the gaze orienting individuals using an algorithm that eliminated head or body features and replaced them with the background (Figure 1). To understand whether the orienting of attention with the head/body cues is transient or sustained, we utilized two different delay periods ( $200 \mathrm{~ms}$ and $500 \mathrm{~ms}$ ) before the target's appearance. We measured the effects of the head, body, or joint direction validity (cueing effect) on accuracy detecting the target person. In addition, we measured how the head/body direction influenced the direction of microsaccades. Previous studies have found that microsaccade rates, latency, and directions are highly correlated with the spatial cueing direction of covert attention (Engbert \& Kliegl, 2003; Hafed \& Clark, 2002; Meyberg, Sinn, et al., 2017; Pastukhov \& Braun, 2010; Poletti et al., 2017). The majority of the microsaccade studies have used static cues such as central arrows (Engbert \& Kliegl, 2003; Meyberg, Sommer, et al., 2017) or simple shapes or flashes present in the periphery (Hafed \& Clark, 2002; Laubrock et al., 2005). Here, we assessed how microsaccades are influenced by the dynamic head, body, and joint orienting and compared their modulation to the influences on behavioral performance.

\section{Material and Methods}

\section{Subjects}

Thirty undergraduate students (age from 18-21, 18 females, 12 males) from the University of California Santa Barabra were recruited as subjects for credits in this experiment. All have normal to corrected-to-normal vision. All participants signed consent forms to participate in the study. The sample size was selected based on previous studies on gaze cues, which usually range from 20-30 (Friesen \& Kingstone, 1998; Palanica \& Itier, 2015; Yokoyama \& Takeda, 2019).

\section{Experimental Setup and Stimuli}

All stimuli were presented at the center of the computer screen, subtending a visual angle of $18.4^{\circ} \times 13.8^{\circ}$ (width $\mathrm{x}$ height). Participants' eyes were $75 \mathrm{~cm}$ from the computer screen with the 
head positioned on a chin rest while watching the videos. Each subject's left eye was tracked by a video-based eye tracker (SR Research Eyelink 1000 plus Desktop Mount) with a sampling rate of $1000 \mathrm{~Hz}$. Subjects' eye movements were calibrated and validated before the experiment. Events in which velocity was higher than $35^{\circ} / \mathrm{s}$ and acceleration exceeded $9500^{\circ} / \mathrm{s}^{2}$ were recorded as saccades. Microsaccades were detected using the method proposed by Engbert and Kliegl (Engbert \& Kliegl, 2003). A microsaccade was defined as intervals longer than $12 \mathrm{~ms}$ and with a velocity above a threshold. The threshold was calculated as a constant $\lambda$ times the estimated standard deviation of microsaccades velocity distribution within each trial. We chose a value of $\lambda=6$ (Engbert \& Kliegl, 2003).

Stimuli consisted of sixty videos (about 3s long) recorded at the University of California Santa Barbara campus across 30 days. The videos were filmed in different settings (classrooms, campus outdoors, student apartments, etc.). In each video, there were between 4-7 people presented. We gave verbal instructions during filming so that multiple people in the video looked simultaneously toward the same person. We first extracted individual frames from original videos. Then we used a manual segmentation of the outline individuals' heads and bodies. To delete target/distractor individuals from the images, we replaced the RGB values of pixels contained by the individual outline in selected frames with the RGB values of those pixels of the immediate background to the individuals. This method allowed us to delete target/distractor individuals from the initial portion of the video frames before the end of the individuals orienting their gaze. Finally, we compiled the processed frames to create videos consisting of: 1 . One to three individuals orienting their gaze, head, and body towards a point in the scene; 2 . the appearance of 3 or 4 individuals after 200 or 500 ms delay after completion of the head/body/gaze. In target-present trials (50\% of all trials), the target person appeared with 2-3 distractors. In 50\% of all target-present trials, the target person appeared at the location where the gaze-orienting individuals looked (valid gaze cue). In the other $50 \%$ of the target-present trials, a distractor person instead of a target person appeared at the location where gaze-orienting individuals looked at (invalid gaze cue) (A1.a-c). In target-absent trials (50\% of all trials), the 3-4 people that were all distractors appeared (A1.d-f). Figure 1 shows 
example frames from videos in which the target person was present with valid cues (see

Appendix 1 for more examples from target-present invalid trials and target-absent trials.

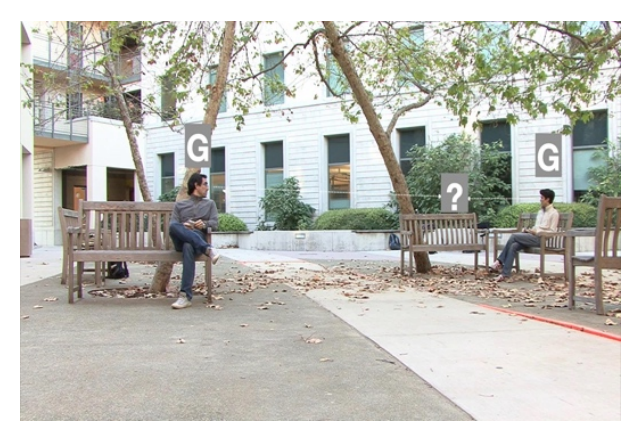

$200 \mathrm{~ms}$ or $500 \mathrm{~ms}$

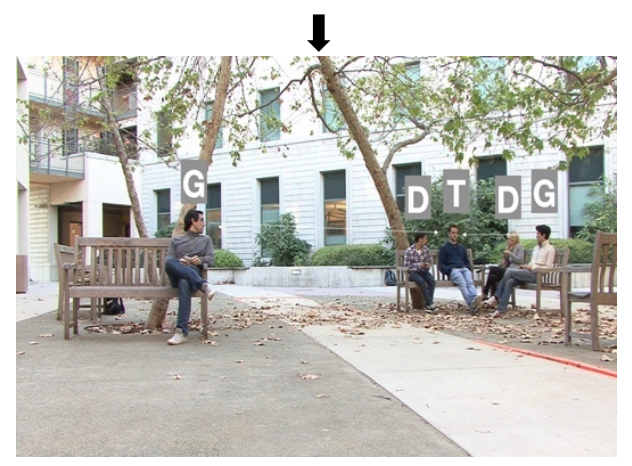

(a) Intact
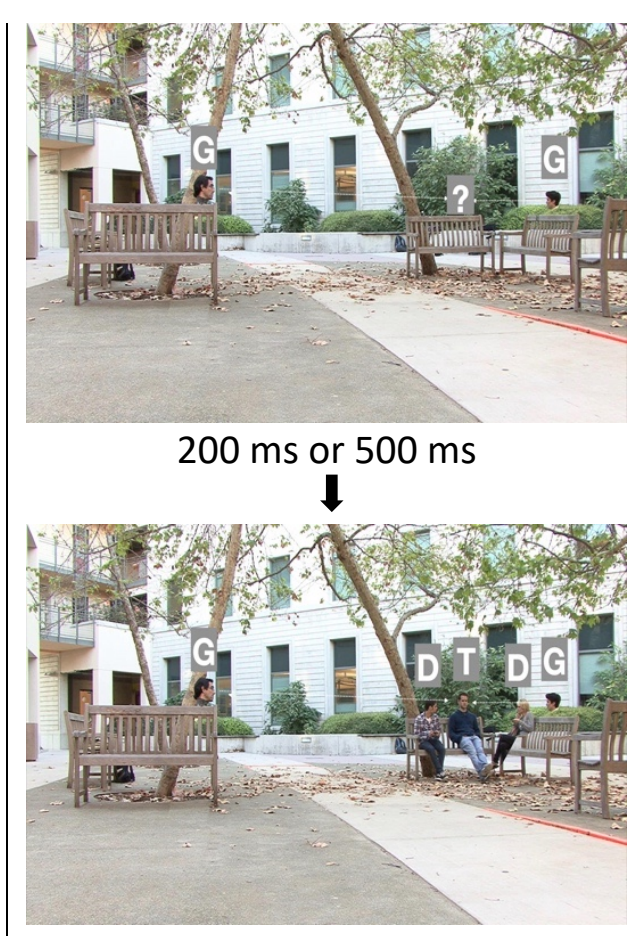

(b) floating heads

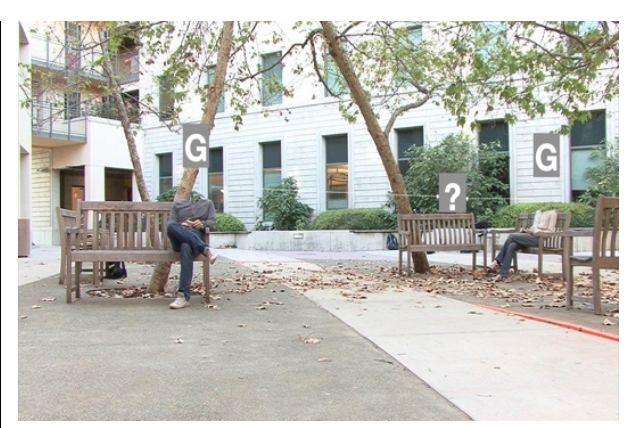

$200 \mathrm{~ms}$ or $500 \mathrm{~ms}$

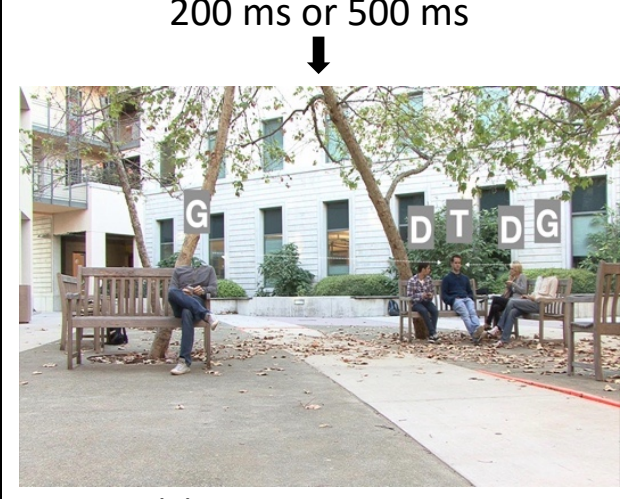

(c) headless bodies

Figure 1. Example frames from videos with valid cues. In the videos, the gaze-orienting individuals (G) look at the target person. After a $200 \mathrm{~ms}$ or $500 \mathrm{~ms}$ delay, the target person ( $\mathrm{T}$ ) and some other distractor people (D) appeared. (a) The intact condition: Gaze-orienting individuals (G) contain head and bodies; (b) the floating heads condition: gaze-orienting individuals $(G)$ have floating heads without bodies; $(c)$ the headless bodies condition: gaze-orienting individuals $(\mathrm{G})$ have only bodies. All the letter annotations and dashed lines that indicate the gaze directions are just for illustration and were not shown in the actual experiment videos.

In addition to the intact condition in which gaze orienting individuals' appeared with heads and bodies, we created videos that selectively deleted gaze orienting individuals' heads or bodies. The target/distractor individuals appearing after the 200 or 500 SOA always contained their entire silhouettes. Thus, there were three experimental conditions: 1. Intact videos, 2. Floating heads videos (gaze-orienting individuals' bodies were invisible), and 3. Headless bodies videos (gaze-orienting individuals' heads were invisible). In all videos, we retained the immediate background behind the erased heads or bodies (See Figure 1). 


\section{Behavioral Task}

Subjects were asked to complete the target detection task while watching videos. Before the practice trials, observers were given unlimited time to familiarize themselves with pictures of the target person in different outfits (See Figure2). Then they completed a practice session with ten videos that were different from the actual experiment videos. Photos of the target person were presented as a reference when they made a response after each video. All participants were able to detect the target above chance after practice.
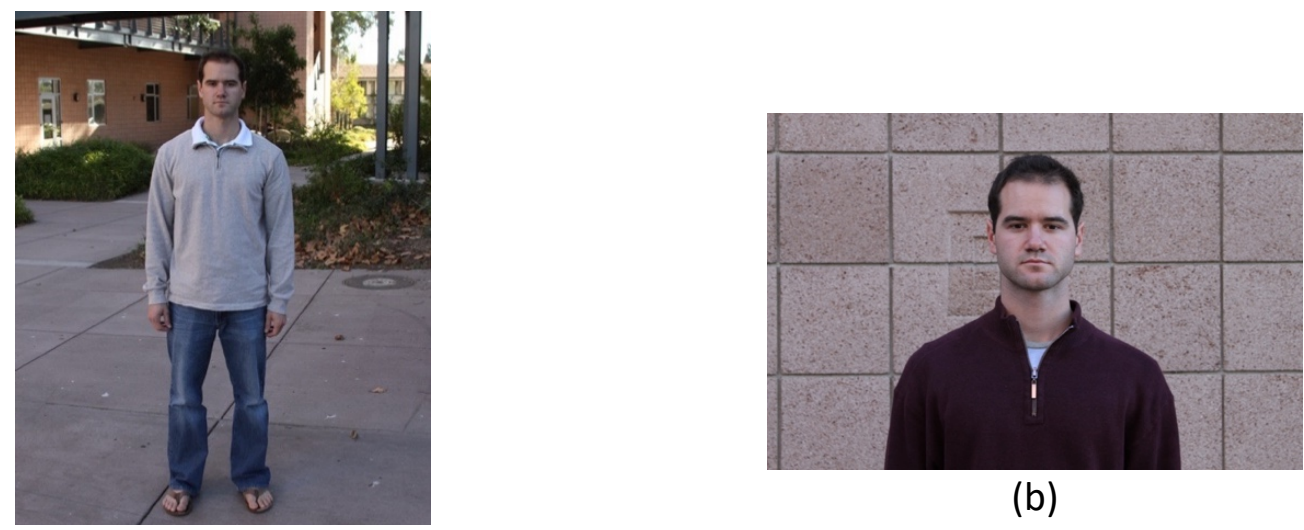

(a)

(b)

Figure 2. The target person's photographs (a). Photo of person in standing position, (b). Photo with a close-up shot. The target person's photos were presented on every response screen.

Participants then completed the main experimental sessions with all three conditions (1.intact; 2.floating heads; 3.headless bodies). During a session, observers completed all three conditions in random order. Videos were presented in blocks for each condition. Within each condition, 60 different videos were presented randomly. In total, each observer finished 60 trials/condition $\mathrm{x}$ 3 conditions $/$ session $\times 2$ sessions $=360$ trials.

Participants first finished a nine-point calibration and validation before experimental sessions started. On each trial, the participants were instructed to fixate a cross at the center of the screen and pressed the space bar to start the trial. They maintained fixation at the central cross 
throughout the presentation of the videos. If the infrared video eye tracker detected an eye movement away from the central fixation of more than $1.5^{\circ}$ visual angle, that trial was aborted. Target and distractors (target-present trials) or only distractors (target-absent trials) were presented for $800 \mathrm{~ms}$, after which a response screen replaced the video. Participants pressed the up arrow or down arrow on the keyboard to indicate if the target person was present or absent (see Figure 3a). Figures $3 b-c$ show the distribution of retinal eccentricities of all gazeorienting individuals and the distribution of viewing angles subtended by their heads (vertical size) across all videos. Figure $3 \mathrm{c}$ also shows the viewing distances from an observer in the real world that would result in the measured subtended angles. We assumed $.24 \mathrm{~m}$ as the average adult head vertical length (the vertical distance from the bottom of the chin to the top of the head) (Lee et al., 2006).

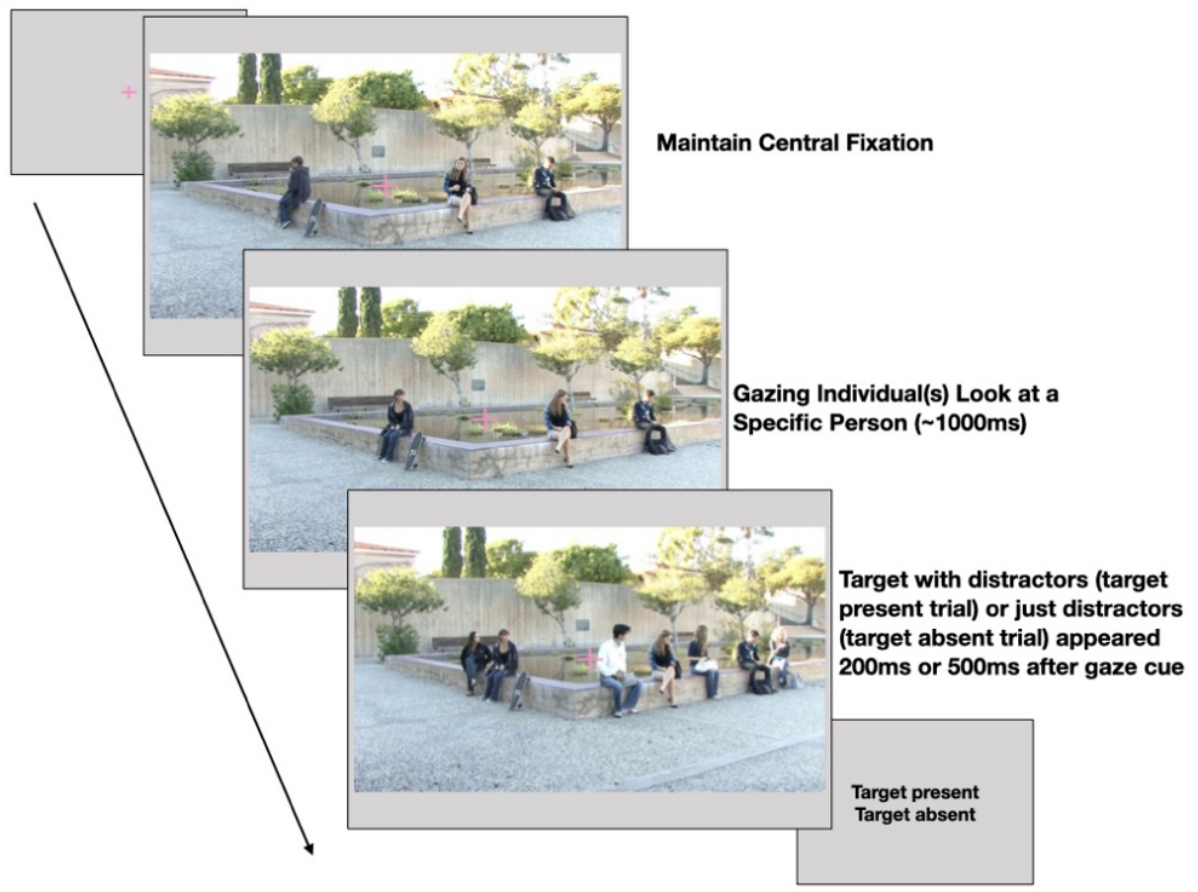

(a) 


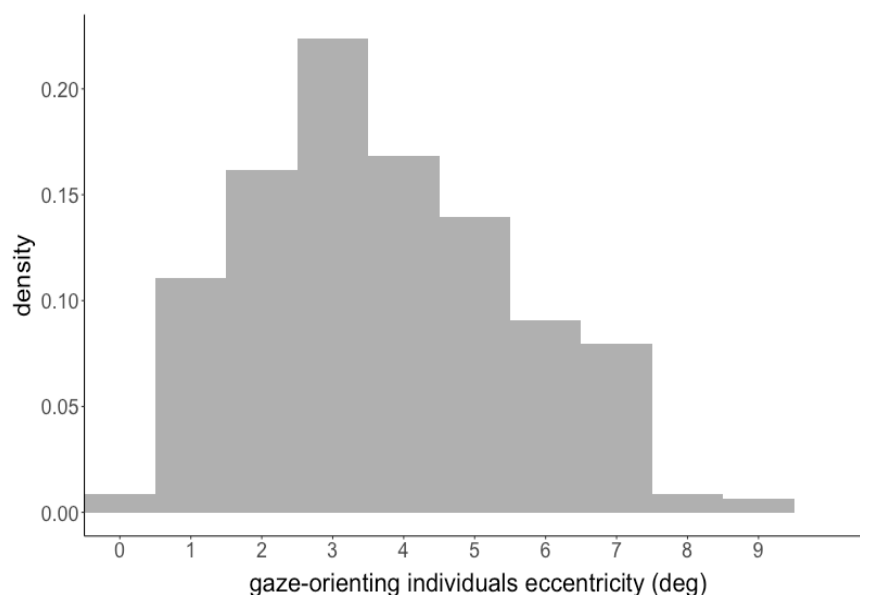

(b)

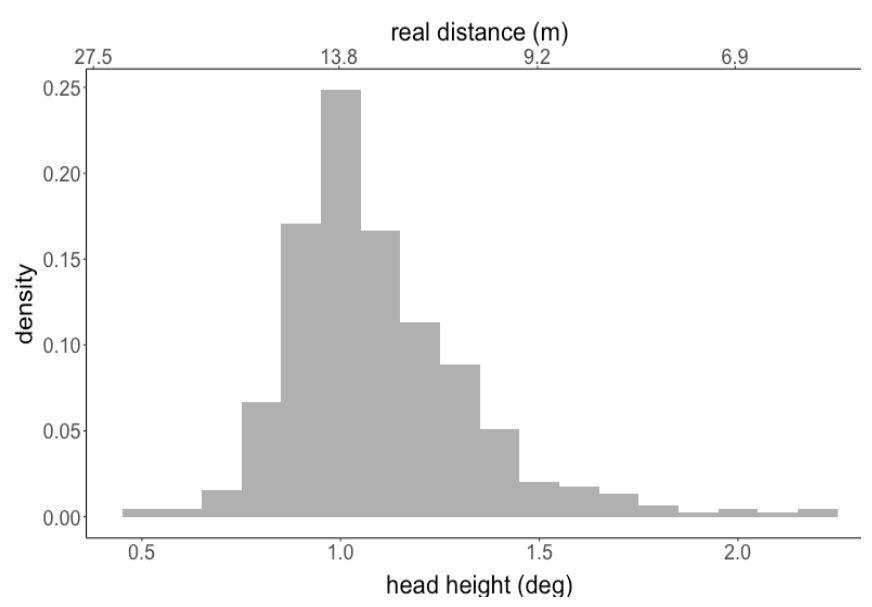

(c)

Figure 3. (a) Timeline for each trial. The participants fixated at the center cross and pressed the space bar to initialize the trial. They maintained fixation at the center cross throughout the video. The trial was aborted if the eye tracker detected a broken fixation (move away from the central fixation by $1.5^{\circ}$ ). The video started with the gaze-orienting individuals looking at a common location of a person not visible during that time period. After $200 \mathrm{~ms}$ or $500 \mathrm{~ms}$ following the end of the gaze looking behavior, other individuals (target-present trials: target person and distractors; targetabsent trials: only distractors) appeared in the video for $800 \mathrm{~ms}$. Participants indicated whether the target person (50\% probability of presence) was present or absent in the video by pressing an upward arrow (present) or a downward arrow (absent). (b).Histogram of retinal eccentricities of the gaze-orienting individuals relative to the central fixation in the movies. (c) Histogram of the vertical size of the gaze-orienting individuals (deg) across all movies. The top-axis is the estimated realworld distances (meters) from the observer that would result in the subtended head vertical angular sizes in the experiment.

\section{Data Analysis}

We first examined the effect of gaze orienting from head and body movement on the subjects' behavioral performance. We used bootstrap techniques to estimate the statistical significance of variations of hit rate, the difference in sensitivity $\Delta d^{\prime}$, and the microsaccades' amplitude (degrees) toward cue directions, and the proportion of microsaccades toward cue direction. Ten thousand bootstrap re-samples for hit rate with valid cues and invalid cues were used to estimate the within-subject difference between valid and invalid trials. For conditions with a $\mathrm{p}$ value close to 0.05 , we used 100,000 bootstrap re-samples to obtain more precise estimates. The procedure was repeated for the three different conditions (intact, floating heads, headless bodies), separated by the length of delay of the onset of the target (200 ms vs. $500 \mathrm{~ms}$ ). We similarly used bootstrap to evaluate the $\Delta \mathrm{d}^{\prime}$, microsaccades' amplitude, and proportion toward 
cue directions. All $p$ values were corrected using a false discovery rate (FDR) to reduce the probability of making a Type I error.

\section{Results}

\section{Gaze cueing effect on perceptual performance}

We first evaluated the influence of the head/body orienting on the performance of detecting the target person. Table 1 shows the hit rate, false alarm rate, and index of detectability d' (Green \& Swets, 1989) for each condition and the statistical significance of the cueing effect. Figure 4 shows the hit rates in the three conditions: the intact, the floating heads, and the headless bodies. In the intact condition, valid head/body cues improved accuracy: a significantly higher hit rate $(\mathrm{hr})$ for both the $200 \mathrm{~ms}$ SOA (valid $\mathrm{hr}=.58 \mathrm{vs}$. invalid $\mathrm{hr}=.47(\mathrm{p}<.001, \mathrm{~d}=.67$, bootstrap for this and all reported tests) and the $500 \mathrm{~ms}$ delay (valid $\mathrm{hr}=.58 \mathrm{vs}$. invalid $\mathrm{hr}=.43$, $p<.001$, Cohen's $d=.77)$. In the floating heads condition, the hit rate was only significant higher for the valid vs. invalid cue trials when the delay was $200 \mathrm{~ms}$ (valid $\mathrm{hr}=.59 \mathrm{vs}$. invalid $\mathrm{hr}=.49$, $p<.001, d=.52$ ), but not with 500 ms delay (valid $h r=.55$ vs. invalid $h r=.51, p=.16, d=.18$ ). Finally, in the headless bodies condition, the cueing effect on hit rate was also found only to be significant in the short delay ( $\mathrm{hr}=.58 \mathrm{vs}$. invalid $\mathrm{hr}=.50, \mathrm{p}=.006, \mathrm{~d}=.49$ ), but not in the $500 \mathrm{~ms}$ delay (valid $h r=.55$ vs. invalid $h r=.50, p=.10, d=.26$ ) (See A2 for same figure for $d^{\prime}$ ). The effect size for the long delay was at least three times larger for the intact condition $(d=.77)$ relative to the floating heads $(d=0.18)$ and the headless bodies conditions $(d=0.26)$.

Figure 5 shows differences in detectability $\left(\Delta d^{\prime}\right)$ across valid and invalid cues for the various conditions. Consistent with hit rate analysis, similar benefits of valid cues were found in the intact condition with both delays $(p<.001, d=.56 ; p<.001, d=.66)$. For the floating heads and the headless bodies conditions, a significant $\Delta d^{\prime}$ was observed with a shorter delay of $200 \mathrm{~ms}$ $(p<.001, d=.56 ; p=.006, d=.47$, respectively) but not the longer delay (floating heads $500 \mathrm{ms,}$ $p=.36, d=.06$; headless bodies $500 \mathrm{~ms}, p=.33, d=.11$ ). The results suggest that the cueing effects elicited by floating heads or headless bodies develop quickly but do not sustain and diminish for the longer SOA delay (500 ms). 


\begin{tabular}{|c|c|c|c|c|c|c|}
\hline \multirow{2}{*}{ Intact } & $\begin{array}{c}\text { Delay } \\
200\end{array}$ & $\begin{array}{c}\text { Cue Validity } \\
\text { valid } \\
\text { invalid }\end{array}$ & $\begin{array}{l}\text { Hit Rate } \\
0.58(.03) \\
0.47(.02) \\
\end{array}$ & $\begin{array}{l}\text { Sensitivity } \\
1.12(.11) \\
0.81(.11) \\
\end{array}$ & $\begin{array}{c}\text { False Alarm } \\
0.22(.02)\end{array}$ & $\begin{array}{l}\text { Cueing Effect } \\
\qquad<<.001\end{array}$ \\
\hline & 500 & $\begin{array}{c}\text { valid } \\
\text { invalid }\end{array}$ & $\begin{array}{l}0.58(.03) \\
0.43(.04)\end{array}$ & $\begin{array}{l}1.13(.16) \\
0.72(.17)\end{array}$ & $0.25(.03)$ & $p<.001$ \\
\hline \multirow{2}{*}{ Floating Heads } & 200 & $\begin{array}{c}\text { valid } \\
\text { invalid }\end{array}$ & $\begin{array}{l}0.59(.04) \\
0.49(.03) \\
\end{array}$ & $\begin{array}{l}1.09(.14) \\
0.81(.14) \\
\end{array}$ & $0.26(.03)$ & $p<.001$ \\
\hline & 500 & $\begin{array}{c}\text { valid } \\
\text { invalid }\end{array}$ & $\begin{array}{l}0.55(.04) \\
0.51(.03) \\
\end{array}$ & $\begin{array}{l}1.06(.17) \\
1.01(.16) \\
\end{array}$ & $0.25(.03)$ & $p=.17$ \\
\hline \multirow{2}{*}{ Headless Bodies } & 200 & $\begin{array}{c}\text { valid } \\
\text { invalid }\end{array}$ & $\begin{array}{l}0.58(.03) \\
0.50(.03) \\
\end{array}$ & $\begin{array}{l}1.15(.11) \\
0.93(.12) \\
\end{array}$ & $0.22(.03)$ & $p=.006$ \\
\hline & 500 & $\begin{array}{c}\text { valid } \\
\text { invalid }\end{array}$ & $\begin{array}{l}0.55(.04) \\
0.50(.03)\end{array}$ & $\begin{array}{l}1.00(.16) \\
0.93(.14)\end{array}$ & $0.25(.03)$ & $p=.08$ \\
\hline
\end{tabular}

Table 1. Mean hit rates, $d^{\prime}$, and false-positive rate, standard errors for each condition in parenthesis, and $\mathrm{p}$-value from bootstrap resampling tests.

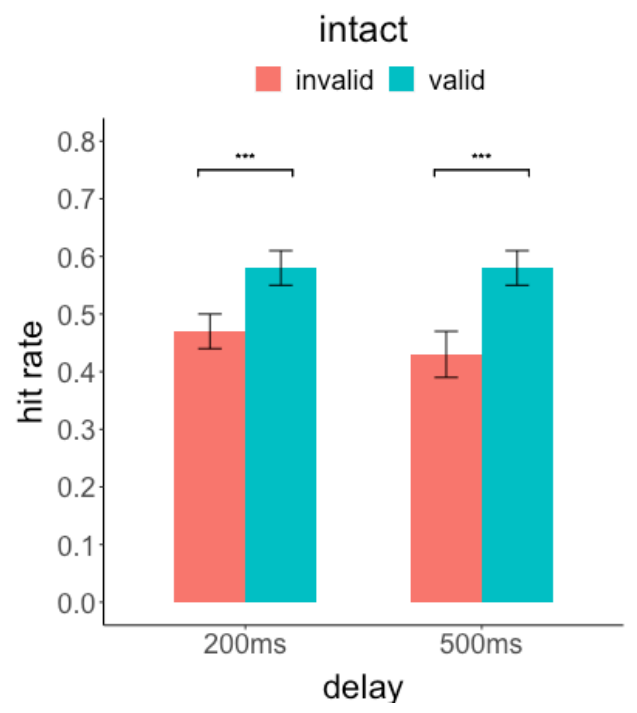

(a)

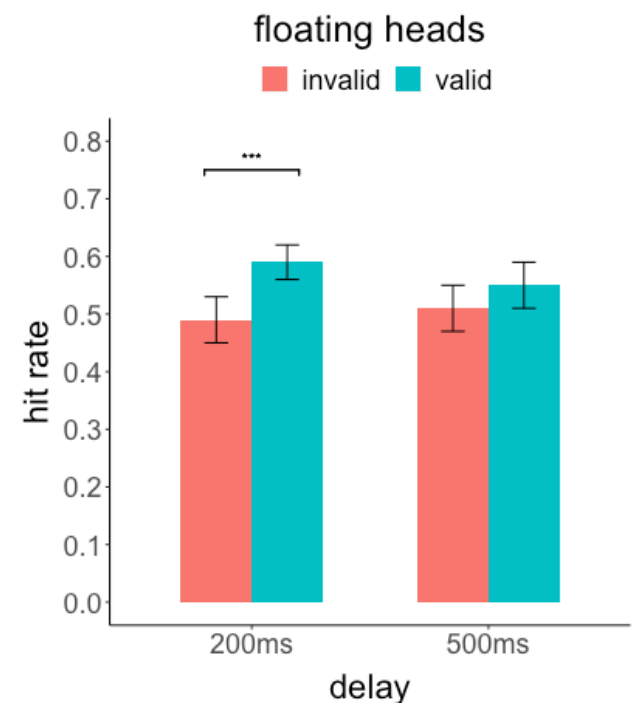

(b)

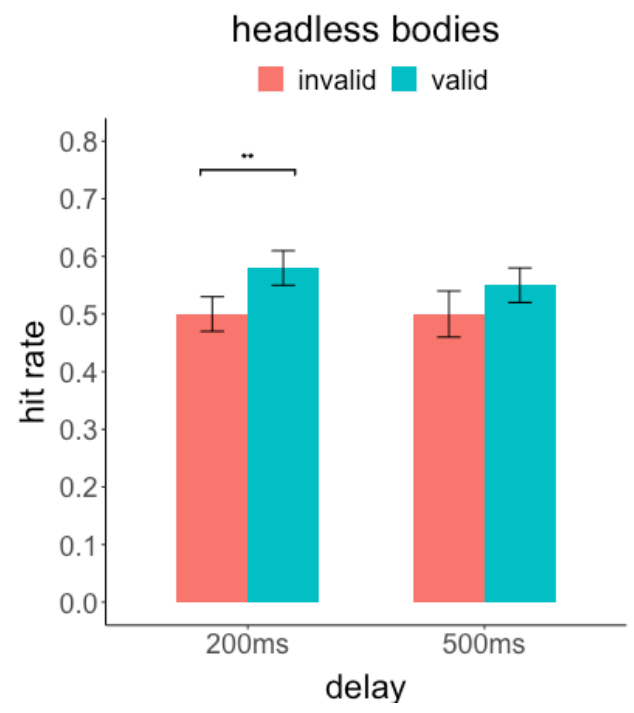

(c)

Figure 4. Hit Rate for cue-valid and invalid trials and two SOA delays (200 ms and $500 \mathrm{~ms}$ ) for the intact condition (a), the floating heads condition (b), the headless bodies condition (c) 


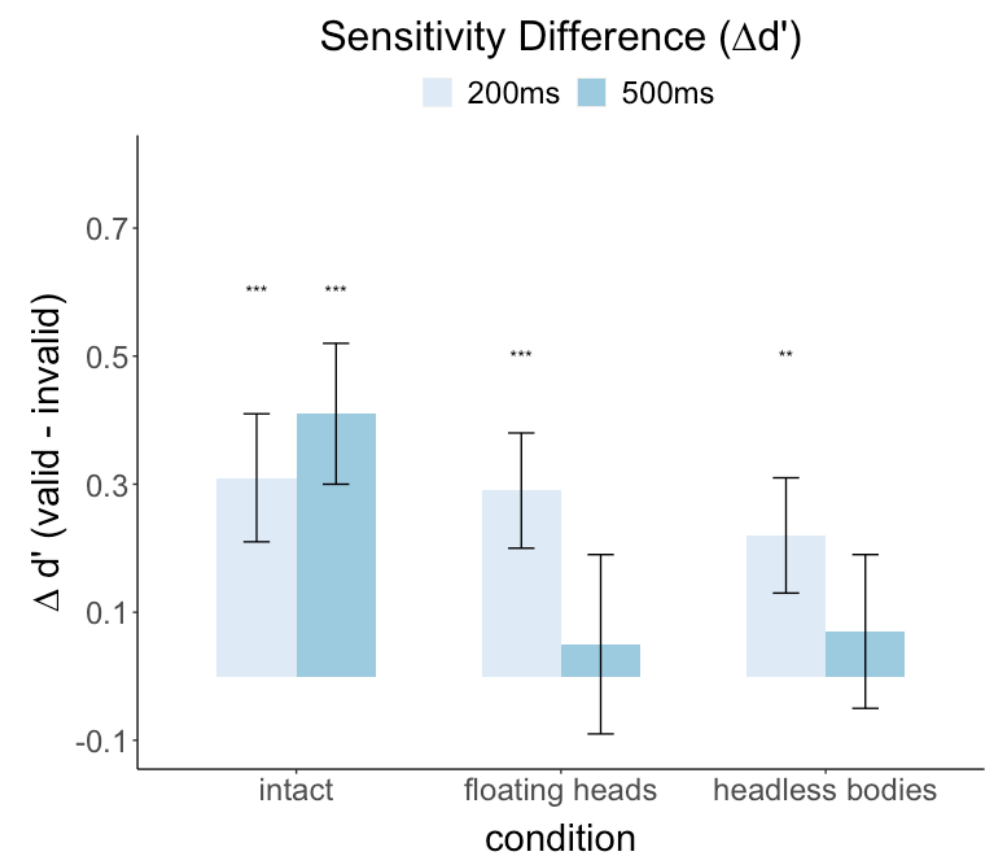

Figure 5. Differences in sensitivity $\left(\Delta d^{\prime}\right)$ across cue-valid and invalid trials for the three conditions and two SOAs.

\section{Cueing Effects on Microsaccade Direction}

We analyzed the amplitude and direction of microsaccades of the time interval before and during the target presentation. Overall the distribution of microsaccade amplitudes was consistent with previous results (Laubrock et al., 2010; Martinez-Conde et al., 2009). Across the entire timecourse, $79.64 \%$ of the microsaccades magnitudes were less than $.5^{\circ}$ visual angle (histogram in Appendix 3). The median microsaccade amplitude was significantly higher when both head and body were presented (intact median $=15.6^{\prime}$ ) than either only heads (floating heads median $=14.9^{\prime}, \chi^{2}=6.06, p=.02$, effect size $\left.\phi=.02\right)(\mathrm{Kim}, 2017)$, or only bodies were present (headless bodies median $=14.2^{\prime}, \chi^{2}=24.29, p<.001, \phi=.04$ ). The median microsaccades amplitude for the floating heads condition was the same as the headless bodies condition $\left(\chi^{2}=\right.$ 3.31, $p=.06, \phi=.01$, Figure A3). 
We plotted heatmaps to show the density of microsaccade directions and amplitudes (Figures 6 $a-d)$ aligned to the gaze-cue direction. Figures $6 a-b$ show the microsaccade density before the presentation of the target/distractors for the $200 \mathrm{~ms}$ and $500 \mathrm{~ms}$ SOAs. Figures $6 \mathrm{c}-\mathrm{d}$ show the density plots $400 \mathrm{~ms}-800 \mathrm{~ms}$ after the presentation onset of the target/distractor separately for valid and invalid cue trials (for $200 \mathrm{~ms}$ and $500 \mathrm{~ms}$ SOA).

To quantify the effect of gaze cueing on microsaccade direction, we calculated the average proportion of microsaccades per trial moving toward the cued direction using a 400ms window. Figure 6e-f shows the proportion of microsaccades toward the cued direction for the intact condition for the $200 \mathrm{~ms}$ and 500 ms conditions (See appendix A4-5 for the floating heads and headless bodies conditions). We separately computed the measure for the time period before and during the presentation of targets/distractors. We found a significant microsaccade direction bias in the valid trials from the intact and the floating heads condition (SOA $200 \mathrm{ms)}$ during the period $400 \mathrm{~ms}-800 \mathrm{~ms}$ after the target/distractors onset. This indicates that the presence of heads in dynamic gaze cueing is necessary to trigger microsaccades toward the cued direction with a short SOA delay. Furthermore, a $200 \mathrm{~ms}$ SOA indicated that the effect of microsaccade direction bias peaked around 600-1000 ms after the dynamic gaze cue completion.

Figure 7 summarizes the proportion of microsaccades toward the cued direction just during the period of $400 \mathrm{~ms}-800 \mathrm{~ms}$ after targets/distractors onset for the three conditions: the intact, the floating heads, and the headless bodies. Table 2 summarizes the proportion of microsaccades and degrees toward the cued direction for the period of $400 \mathrm{~ms}-800 \mathrm{~ms}$ after targets/distractors onset: valid vs. invalid trials for the two SOAs and three conditions (intact, floating heads, headless bodies). Consistent with the behavioral performance (Figure 4), we found a significant bias towards the cue direction for the valid trials in the intact silhouettes for both 200 ms and $500 \mathrm{~ms}(200 \mathrm{~ms} \mathrm{p}=.005, \mathrm{~d}=.75 ; 500 \mathrm{~ms} \mathrm{p}=.047, \mathrm{~d}=.52$. We also found a significant bias towards the cue direction for the valid trials for floating heads condition only with an SOA of $200 \mathrm{~ms}$ $(p=.03, d=.58$, see Table 2 \& Figure 7$)$ but not with SOA of $500 \mathrm{~ms}(p=.19, d=.24)$. There were no 
significant effects on microsaccades towards the valid cue direction for the headless bodies condition for either SOAs (200 ms: $p=.10, d=.40 ; 500$ ms: $p=.46, d=.05$ ). We also quantified the average microsaccades' degrees toward the cued direction for valid and invalid trials over time and summarized the result in table2 (See also appendix A6,A7).

The sustained influence of the joint presence of head and body in orienting attention is also shown for the $500 \mathrm{~ms}$ SOA in the trend of a microsaccade bias even for invalid cue trials (Figure 7a). This trend is not present for the $500 \mathrm{~ms}$ SOA invalid cue trials of the floating heads or headless bodies conditions (although bootstrap tests with FDR correction did not reach statistical significance when comparing intact vs. the average of the two other conditions, $p=.057, d=.40)$. 


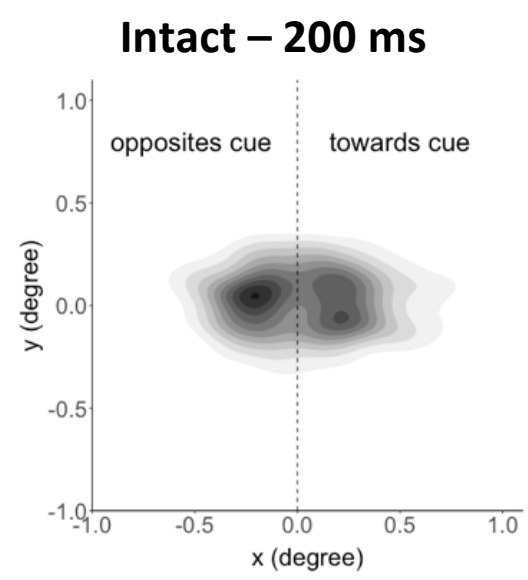

(a) Before Presentation

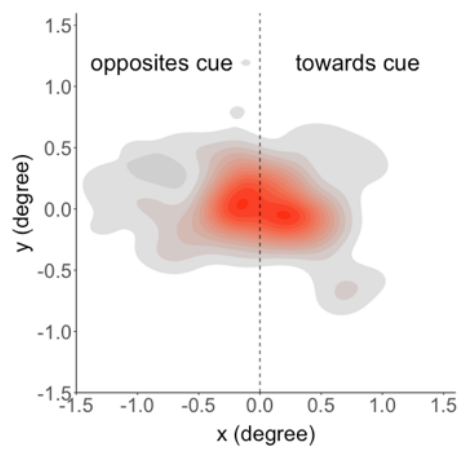

Invalid

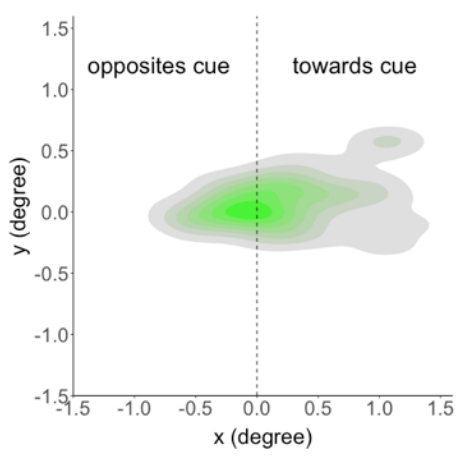

valid

(c) 400-800ms after Target/Distractor(s) Presentation

$\rightarrow$ all $\rightarrow$ valid cue $\rightarrow$ invalid cue

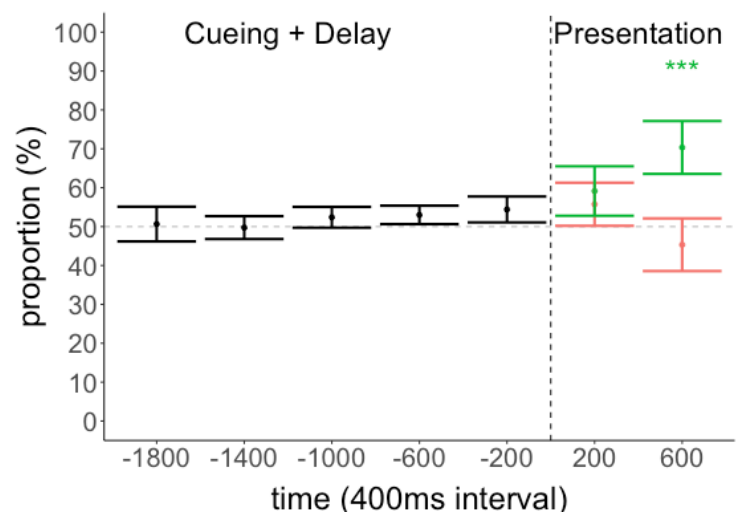

(e)

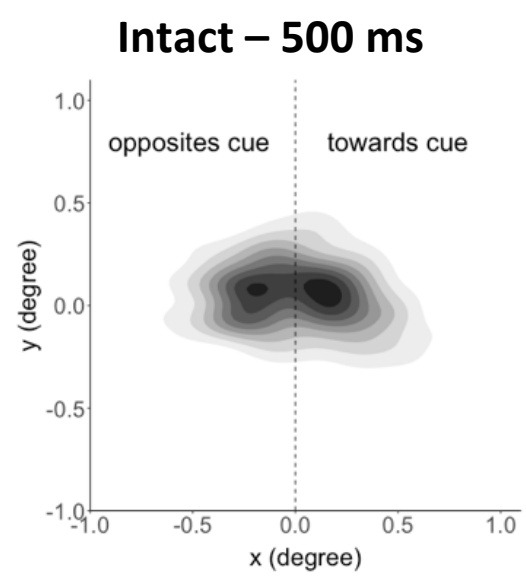

(b) Before Presentation

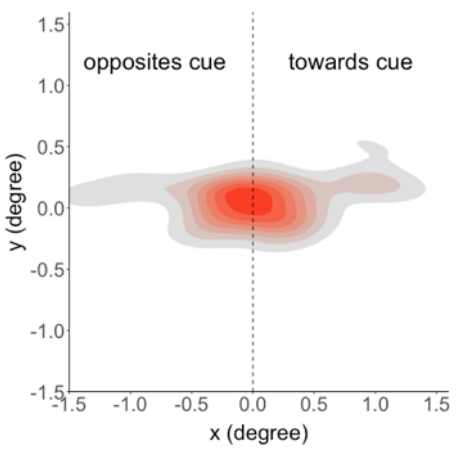

Invalid

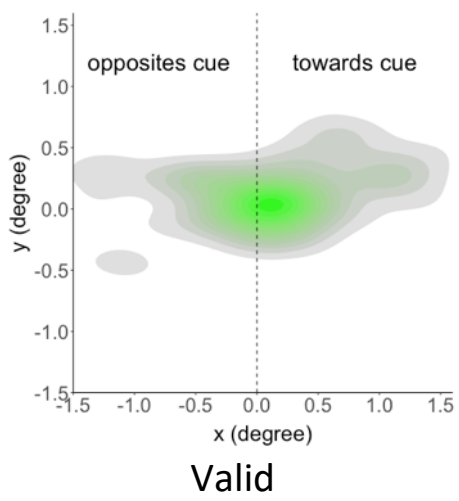

(d) 400-800ms after Target/Distractor(s) Presentation

- all - valid cue - invalid cue

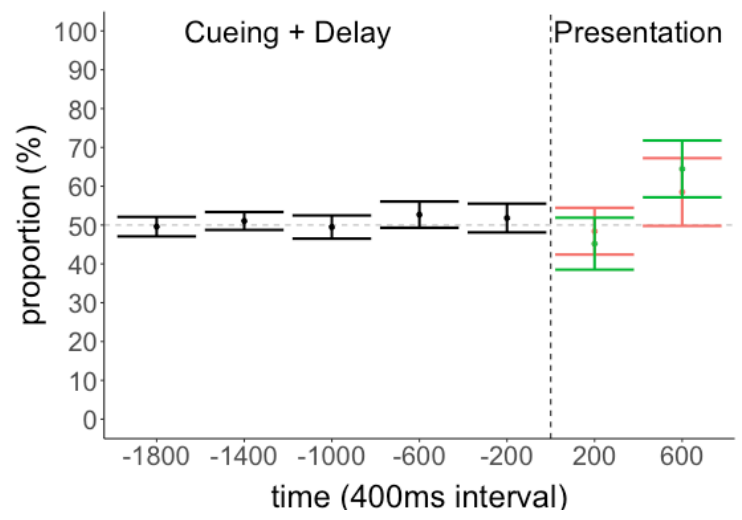

(f)

Figure 6. Microsaccades for the intact condition for $200 \mathrm{~ms}$ and 500ms SOA (a)-(b) Heatmaps of microsaccades for the period before the target/distractors presentation with black and white colors (a darker color corresponds to higher density). (c)-(d) Heatmaps of microsaccades during 400-800 ms after target/distractor presentation (red color for invalid trials, green color for valid trials). (e)-(f) Proportion of microsaccades that moved toward the cued direction as a function of time for intact condition $200 \mathrm{~ms}$ and $500 \mathrm{~ms}$ delay over time. The x-axis indicates the temporal midpoint of the time window (200 represents the time window $0-400 \mathrm{~ms}$, etc.). Time is lined up with respect to target and distractor presentation. The $y$-axis is the mean proportion of microsaccades that moved toward the cue direction. 


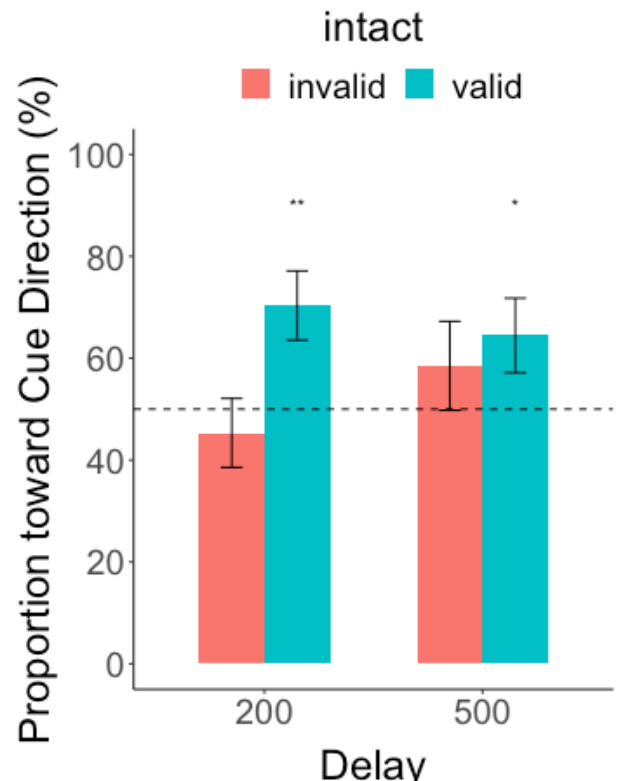

(a)

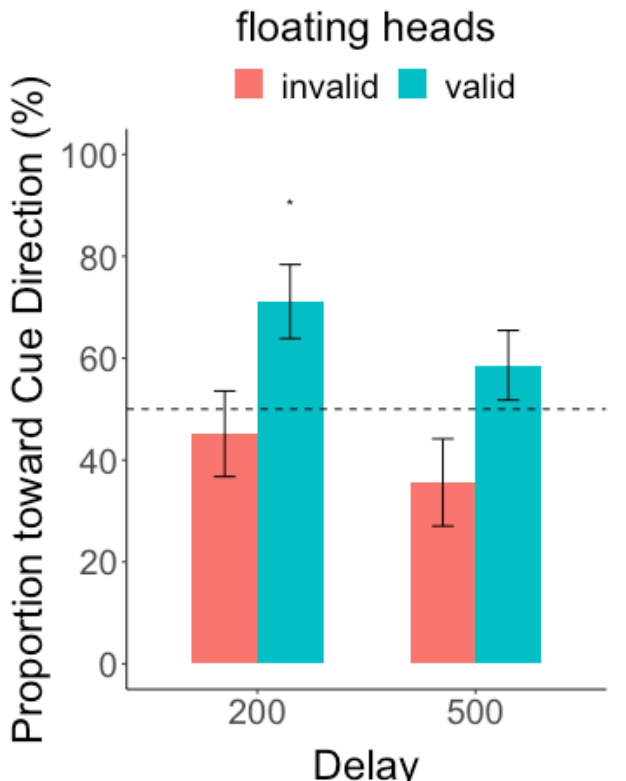

(b)

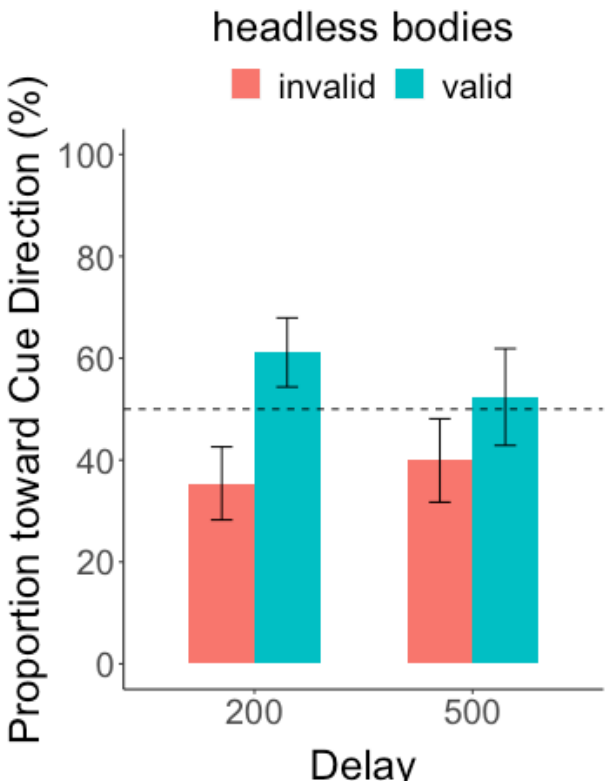

(c)

Figure 7. The proportion of microsaccades that moved toward the cue direction within $400 \mathrm{~ms}-800 \mathrm{~ms}$ after target/distractors presentation onset for intact condition (a), floating heads condition (b), and headless bodies condition (c). Results are shown for the two SOAs or delays.

\begin{tabular}{|c|c|c|c|c|}
\hline Condition & SOA & Cue Validity & $\begin{array}{l}\text { Proportion toward } \\
\text { Cue direction }\end{array}$ & $\begin{array}{l}\text { Degree toward } \\
\text { Cue direction }\end{array}$ \\
\hline \multirow{2}{*}{ Intact } & 200 & $\begin{array}{l}\text { valid } \\
\text { invalid }\end{array}$ & $\begin{array}{c}70.34 \%(p=.005) \\
45.33 \%(p=.30)\end{array}$ & $\begin{array}{l}.23^{\circ}(p=.001) \\
-.13^{\circ}(p=.37)\end{array}$ \\
\hline & 500 & $\begin{array}{c}\text { valid } \\
\text { invalid }\end{array}$ & $\begin{array}{c}64.46 \%(p=.047) \\
58.51 \%(p=.21)\end{array}$ & $\begin{array}{l}.05^{\circ}(p=.27) \\
.02^{\circ}(p=.37)\end{array}$ \\
\hline \multirow{2}{*}{ Floating Heads } & 200 & $\begin{array}{c}\text { valid } \\
\text { invalid }\end{array}$ & $\begin{array}{l}71.12 \%(p=.03) \\
45.16 \%(p=.49)\end{array}$ & $\begin{array}{l}.20^{\circ}(p=.002) \\
-.09^{\circ}(p=.80)\end{array}$ \\
\hline & 500 & $\begin{array}{c}\text { valid } \\
\text { invalid }\end{array}$ & $\begin{array}{l}58.61 \%(p=.19) \\
35.61 \%(p=.13)\end{array}$ & $\begin{array}{r}.10^{\circ}(p=.06) \\
-.12^{\circ}(p=.80)\end{array}$ \\
\hline \multirow{2}{*}{ Headless Bodies } & 200 & $\begin{array}{c}\text { valid } \\
\text { invalid }\end{array}$ & $\begin{array}{l}61.14 \%(p=.10) \\
35.42 \%(p=.13)\end{array}$ & $\begin{array}{c}.03^{\circ}(p=.31) \\
-.06^{\circ}(p=.99)\end{array}$ \\
\hline & 500 & $\begin{array}{l}\text { valid } \\
\text { invalid }\end{array}$ & $\begin{array}{l}52.38 \%(p=.46) \\
39.92 \%(p=.16)\end{array}$ & $\begin{array}{l}.08^{\circ}(p=.31) \\
-.06^{\circ}(p=.99)\end{array}$ \\
\hline
\end{tabular}

Table 2 . The average microsaccades visual angle degrees toward the cue direction 


\section{Discussion}

Our goal was to measure how gaze, heads and bodies during natural behaviors contribute to orienting attention in an ecological search task with dynamic scenes. The majority of previous studies with simpler drawings, point-light stimuli (Shi et al., 2010), and simple videos of heads suggest that gaze, head, and body direction result in cueing effects that persist up to $600 \mathrm{~ms}$ or beyond. However, in all of these studies, the gaze/posture cue is presented foveally and subtends a large viewing angle. Our study found a different result: heads and bodies oriented attention in a sustained manner until 500 ms only when presented jointly. The separate presentations of the head or body led to more transient cueing effects, which diminished with the 500 ms delay. What factors might explain the discrepancy from previous results? We aimed to simulate a broader range of scenarios in the real world where the observer views a scene from medium to large distances and gazing individuals appear at various retinal eccentricities. Previous studies have shown that at distances beyond 4-5 degrees, inferences about gaze direction from eye orientation are highly degraded (Loomis et al., 2008), and cueing gaze effects are reduced (Yokoyama \& Takeda, 2019). As retinal eccentricity increases, head orientation plays a larger role as information about eye orientation becomes less accessible due to crowding in the visual periphery (Florey et al., 2015). The more difficult access to eye orientation might explain the difference between the current and previous results. In the present study, we could not isolate the specific relationship between the eccentricity of gazeorienting people and observer performance because each video presented multiple individuals at different eccentricities.

We integrate our findings into the current literature as follows. When the eyes are presented foveally and clearly visible, gaze direction generates a sustained orienting of attention. In situations in which the individuals are more distant and away from the point of fixation, sustained attention requires the presence of whole silhouettes of the gazing individuals. Where might the integration of the various gaze, head, and body cues occur? Neuropsychological and neuroimaging studies point to the superior temporal sulcus (STS) as playing an important role in integrating multiple cues to develop social perception. STS cells are sensitive to images of the 
face, gaze direction, mouth movement, head orientation (Perrett et al., 1985, 1992), biological motion such as eyes, mouth, and body movements (Bonda et al., 1996; Grossman et al., 2000), and goal-oriented hand and body movements that help infer another person's attention (Bonda et al., 1996; Jellema et al., 2000; Perrett et al., 1989). Humans and monkeys with damaged STS regions show difficulty identifying others' faces, gaze direction, and intention (Campbell et al., 1990; Heywood \& Cowey, 1992).

Our study also investigated the effect of the direction of the gazing individuals on microsaccades. Previous studies found microsaccades direction biases toward endogenous cue (e.g., central cue) direction around $200 \mathrm{~ms}-400 \mathrm{~ms}$ after cue onset during a saccade rate rebound period (Engbert \& Kliegl, 2003). However, with exogenous cues (peripheral flash), microsaccade directions were found to be biased toward cue direction around $20 \mathrm{~ms}-200 \mathrm{~ms}$ after cue onset, then shift back to the opposite direction around 600ms-800ms (Laubrock et al., 2005; Rolfs et al., 2004). We found an amplitude of the microsaccade bias toward cue direction $\left(\sim 0.2^{\circ}\right)$ that is higher than previous studies (less than $0.05^{\circ}$ ) (Meyberg, Sommer, et al., 2017). Yet, our current results differ from previous studies in important ways. The dynamic cueing by the combination of heads and bodies resulted in a microsaccade direction bias toward the cue direction at a much later time period, around 800-1000 ms after the completion of the movement of the gazing individuals. The longer delays might be related to: 1 . The cues in our study were not static but dynamic social cues which develop throughout the $2 \mathrm{~s}$ of the video. 2 . The dynamic cues were not at the center but in the periphery. 3. We presented multiple gazecueing individuals. Thus, it might take a longer time to integrate peripheral information across the various gazing individuals. The microsaccades also showed more frequent movements towards the cue direction in valid vs. invalid trials for the intact condition but not for the headless bodies condition.

Our study found consistency between the orienting of covert attention as measured by the influence of the cue on perceptual performance and by the microsaccade shifts. Microsaccade direction biases and cue validity benefits on behavioral performance were present for an SOA 
of 200 ms after cue presentation for the intact and floating heads conditions. Only the intact condition showed sustained effect with an SOA of 500 ms for both behavioral performance and microsaccade direction bias. The headless bodies showed a higher behavioral performance but did not show a significant microsaccade direction bias with an SOA of $200 \mathrm{~ms}$. Together, the results are consistent with previous evidence and interpretations suggesting that microsaccades precede or reflect shifts of covert attention (Engbert \& Kliegl, 2003; Hafed \& Clark, 2002; Laubrock et al., 2010; Yuval-Greenberg et al., 2014).

Finally, our study provides an extension of previous studies to more ecologically valid tasks. A majority of studies measure facilitation in the gaze direction for the detection of very simple stimuli. Our study demonstrates the role of gaze, head, and body movements in orienting attention and its impact on decision accuracy for a complex and real-world task such as a person search in cluttered scenes. The experimental framework and publically available videos can be potentially used to study individuals with deficits in social attention using a paradigm that better reflects real-world scenes and tasks. 


\section{Declarations}

\section{Funding.}

The research was supported by the Institute for Collaborative Biotechnologies through contract W911NF-19-D-001 and cooperative agreement W911NF-19-2-0026, both from the US Army Research Office. The views and conclusions contained in this document are those of the authors and should not be interpreted as representing the official policies, either expressed or implied, of the US Government. The US Government is authorized to reproduce and distribute reprints for Government purposes notwithstanding any copyright notation herein.

\section{Conflicts of Interest}

The authors have no conflicts of interest to declare that are relevant to the content of this article.

\section{Ethics Approval}

Approval was obtained from the ethics committee of the University of California Santa Barbara. Consent to participate

Informed consent was obtained from all individual participants in the study.

\section{Availability of data and materials}

The video stimuli and codes are available from the corresponding author Nicole X. Han at xhan01@ucsb.edu on request.

\section{Authors' contributions}

NXH and MPE conceived and designed the studies and determined the data analysis. NXH implemented the experiment, collected and analyzed the data. NXH and MPE both wrote the manuscript. Both authors approved the final version of the manuscript for submission. 


\section{References}

Azarian, B., Buzzell, G. A., Esser, E. G., Dornstauder, A., \& Peterson, M. S. (2017). Averted body postures facilitate orienting of the eyes. Acta Psychologica, 175, 28-32. https://doi.org/10.1016/j.actpsy.2017.02.006

Bayliss, A. P., di Pellegrino, G., \& Tipper, S. P. (2004). Orienting of attention via observed eye gaze is head-centred. Cognition, 94(1), B1-10. https://doi.org/10.1016/j.cognition.2004.05.002

Bonda, E., Petrides, M., Ostry, D., \& Evans, A. (1996). Specific involvement of human parietal systems and the amygdala in the perception of biological motion. The Journal of Neuroscience: The Official Journal of the Society for Neuroscience, 16(11), 3737-3744.

Bräuer, J., Call, J., \& Tomasello, M. (2005). All Great Ape Species Follow Gaze to Distant Locations and Around Barriers. Journal of Comparative Psychology, 119(2), 145-154. https://doi.org/10.1037/0735-7036.119.2.145

Brooks, R., \& Meltzoff, A. N. (2005). The development of gaze following and its relation to language. Developmental Science, 8(6), 535-543. https://doi.org/10.1111/j.14677687.2005.00445.x

Bugnyar, T., Stöwe, M., \& Heinrich, B. (2004). Ravens, Corvus corax, follow gaze direction of humans around obstacles. Proceedings. Biological Sciences, 271(1546), 1331-1336. https://doi.org/10.1098/rspb.2004.2738

Campbell, R., Heywood, C. A., Cowey, A., Regard, M., \& Landis, T. (1990). Sensitivity to eye gaze in prosopagnosic patients and monkeys with superior temporal sulcus ablation. Neuropsychologia, 28(11), 1123-1142. https://doi.org/10.1016/0028-3932(90)90050-X 
Carrasco, M. (2006). Covert attention increases contrast sensitivity: Psychophysical, neurophysiological and neuroimaging studies. Progress in Brain Research, 154, 33-70. https://doi.org/10.1016/S0079-6123(06)54003-8

Carrasco, M. (2011). Visual attention: The past 25 years. Vision Research, 51(13), 1484-1525. https://doi.org/10.1016/j.visres.2011.04.012

Castelhano, M. S., \& Heaven, C. (2011). Scene context influences without scene gist: Eye movements guided by spatial associations in visual search. Psychonomic Bulletin \& Review. https://doi.org/10.3758/s13423-011-0107-8

Cheal, M., \& Lyon, D. R. (1991). Central and peripheral precuing of forced-choice discrimination. The Quarterly Journal of Experimental Psychology Section A, 43(4), 859-880. https://doi.org/10.1080/14640749108400960

Corbetta, M., \& Shulman, G. L. (2002). Control of goal-directed and stimulus-driven attention in the brain. Nature Reviews. Neuroscience, 3(3), 201-215. https://doi.org/10.1038/nrn755

Dosher, B. A., \& Lu, Z. L. (2000). Mechanisms of perceptual attention in precuing of location. Vision Research, 40(10), 1269-1292.

Driver, J., Davis, G., Ricciardelli, P., Kidd, P., Maxwell, E., \& Baron-cohen, S. (1999). Gaze perception triggers reflexive visuospatial orienting. Visual Cognition, 509-540.

Eckstein, M. (2017). Probabilistic Computations for Attention, Eye Movements, and Search. Annu. Rev. Vis. Sci., 3:18.1-18.24. 
Eckstein, M. P., Drescher, B. A., \& Shimozaki, S. S. (2006). Attentional Cues in Real Scenes, Saccadic Targeting, and Bayesian Priors. Psychological Science, 17(11), 973-980. https://doi.org/10.1111/j.1467-9280.2006.01815.x

Eckstein, M. P., Mack, S. C., Liston, D. B., Bogush, L., Menzel, R., \& Krauzlis, R. J. (2013). Rethinking human visual attention: Spatial cueing effects and optimality of decisions by honeybees, monkeys and humans. Vision Research. https://doi.org/10.1016/j.visres.2012.12.011

Eckstein, M. P., Pham, B. T., \& Shimozaki, S. S. (2004). The footprints of visual attention during search with $100 \%$ valid and $100 \%$ invalid cues. Vision Research, 44(12), 1193-1207. https://doi.org/10.1016/j.visres.2003.10.026

Eckstein, M. P., Shimozaki, S. S., \& Abbey, C. K. (2002). The footprints of visual attention in the Posner cueing paradigm revealed by classification images. Journal of Vision, 2(1), 25-45. https://doi.org/10:1167/2.1.3

Egeth, H. E., \& Yantis, S. (1997). VISUAL ATTENTION: Control, Representation, and Time Course. Annual Review of Psychology, 48(1), 269-297. https://doi.org/10.1146/annurev.psych.48.1.269

Emery, N. J. (2000). The eyes have it: The neuroethology, function and evolution of social gaze. Neuroscience \& Biobehavioral Reviews, 24(6), 581-604. https://doi.org/16/S01497634(00)00025-7

Engbert, R., \& Kliegl, R. (2003). Microsaccades uncover the orientation of covert attention. Vision Research, 43(9), 1035-1045. https://doi.org/10.1016/S0042-6989(03)00084-1 
Florey, J., Clifford, C. W. G., Dakin, S. C., \& Mareschal, I. (2015). Peripheral processing of gaze. Journal of Experimental Psychology: Human Perception and Performance, 41(4), 10841094. https://doi.org/10.1037/xhp0000068

Friesen, C. K., \& Kingstone, A. (1998). The eyes have it! Reflexive orienting is triggered by nonpredictive gaze. Psychonomic Bulletin \& Review, 5(3), 490-495. https://doi.org/10.3758/BF03208827

Friesen, C. K., Ristic, J., \& Kingstone, A. (2004). Attentional effects of counterpredictive gaze and arrow cues. Journal of Experimental Psychology. Human Perception and Performance, 30(2), 319-329. https://doi.org/10.1037/0096-1523.30.2.319

Frischen, A., Bayliss, A. P., \& Tipper, S. P. (2007). Gaze cueing of attention: Visual attention, social cognition, and individual differences. Psychological Bulletin, 133(4), 694-724. https://doi.org/10.1037/0033-2909.133.4.694

Gandhi, S. P., Heeger, D. J., \& Boynton, G. M. (1999). Spatial attention affects brain activity in human primary visual cortex. Proceedings of the National Academy of Sciences, 96(6), 3314-3319. https://doi.org/10.1073/pnas.96.6.3314

Garcia, J. O., Srinivasan, R., \& Serences, J. T. (2013). Near-Real-Time Feature-Selective Modulations in Human Cortex. Current Biology, 23(6), 515-522. https://doi.org/10.1016/j.cub.2013.02.013

Giesbrecht, B., Woldorff, M. G., Song, A. W., \& Mangun, G. R. (2003). Neural mechanisms of top-down control during spatial and feature attention. Neurolmage, 19(3), 496-512.

Green, D. M., \& Swets, J. A. (1989). Signal Detection Theory and Psychophysics. Peninsula Pub. 
Grossman, E., Donnelly, M., Price, R., Pickens, D., Morgan, V., Neighbor, G., \& Blake, R. (2000). Brain Areas Involved in Perception of Biological Motion. Journal of Cognitive Neuroscience, 12(5), 711-720. https://doi.org/10.1162/089892900562417

Hafed, Z. M., \& Clark, J. J. (2002). Microsaccades as an overt measure of covert attention shifts. Vision Research, 42(22), 2533-2545. https://doi.org/10.1016/S0042-6989(02)00263-8 Hermens, F., \& Walker, R. (2012). Do you look where I look? Attention shifts and response preparation following dynamic social cues. Journal of Eye Movement Research, 5(5), Article 5. https://doi.org/10.16910/jemr.5.5.5

Hessels, R. S. (2020). How does gaze to faces support face-to-face interaction? A review and perspective. Psychonomic Bulletin \& Review, 27(5), 856-881. https://doi.org/10.3758/s13423-020-01715-w

Heywood, C., \& Cowey, A. (1992). The Role of the 'Face-Cell' Area in the Discrimination and Recognition of Faces by Monkeys [and Discussion]. Philosophical Transactions of the Royal Society of London. Series B, Biological Sciences, 335, 31-37; discussion 37. https://doi.org/10.1098/rstb.1992.0004

Hietanen, J. K. (1999). Does your gaze direction and head orientation shift my visual attention? Neuroreport, 10(16), 3443-3447. https://doi.org/10.1097/00001756-199911080-00033 Hietanen, J. K. (2002). Social attention orienting integrates visual information from head and body orientation. Psychological Research, 66(3), 174-179. https://doi.org/10.1007/s00426-002-0091-8

Jellema, T., Baker, C. I., Wicker, B., \& Perrett, D. I. (2000). Neural representation for the perception of the intentionality of actions. Brain Cogn 44:280-302. 
Jonides, J., \& JONIDES, J. (1981). Voluntary versus automatic control over the mind's eye's movement. http://wexler.free.fr/library/files/jonides\%20(1981)\%20voluntary\%20versus\%20automa tic\%20control\%20over\%20the\%20mind's\%20eye's\%20movements.pdf

Kim, H.-Y. (2017). Statistical notes for clinical researchers: Chi-squared test and Fisher's exact test. Restorative Dentistry \& Endodontics, 42(2), 152-155. https://doi.org/10.5395/rde.2017.42.2.152

Kingstone, A., Friesen, C., \& Gazzaniga, M. (2000). Reflexive Joint Attention Depends on Lateralized Cortical Connections. Psychological Science, 11, 159-166. https://doi.org/10.1111/1467-9280.00232

Kleinke, C. L. (1986). Gaze and eye contact: A research review. Psychological Bulletin, 100(1), 78-100. https://doi.org/10.1037/0033-2909.100.1.78

Koehler, K., \& Eckstein, M. (2017). Beyond Scene Gist: Objects guide search more than backgrounds. Journal of Experimental Psychology. Human Perception and Performance.

Kuhn, G., \& Tipples, J. (2011). Increased gaze following for fearful faces. It depends on what you're looking for! Psychonomic Bulletin \& Review, 18(1), 89-95. https://doi.org/10.3758/s13423-010-0033-1

Langton, S. R. H., Honeyman, H., \& Tessler, E. (2004). The influence of head contour and nose angle on the perception of eye-gaze direction. Perception \& Psychophysics, 66(5), 752771. https://doi.org/10.3758/BF03194970

Laubrock, J., Engbert, R., \& Kliegl, R. (2005). Microsaccade dynamics during covert attention. Vision Research, 45(6), 721-730. https://doi.org/10.1016/j.visres.2004.09.029 
Laubrock, J., Kliegl, R., Rolfs, M., \& Engbert, R. (2010). When do microsaccades follow spatial attention? Attention, Perception, \& Psychophysics, 72(3), 683-694. https://doi.org/10.3758/APP.72.3.683

Lee, J.-H., Hwang Shin, S.-J., \& Istook, C. L. (2006). Analysis of Human Head Shapes in the United States. International Journal of Human Ecology, 7(1), 77-83.

Loomis, J. M., Kelly, J. W., Pusch, M., Bailenson, J. N., \& Beall, A. C. (2008). Psychophysics of perceiving eye-gaze and head direction with peripheral vision: Implications for the dynamics of eye-gaze behavior. Perception, 37(9), 1443-1457. https://doi.org/10.1068/p5896

Luck, S. J., Hillyard, S. A., Mouloua, M., Woldorff, M. G., Clark, V. P., \& Hawkins, H. L. (1994). Effects of spatial cuing on luminance detectability: Psychophysical and electrophysiological evidence for early selection. Journal of Experimental Psychology: Human Perception and Performance, 20(4), 887-904. https://doi.org/10.1037/00961523.20.4.887

Malcolm, G. L., \& Henderson, J. M. (2010). Combining top-down processes to guide eye movements during real-world scene search. Journal of Vision, 10(2), 4-4. https://doi.org/10.1167/10.2.4

Mareschal, I., Calder, A. J., Dadds, M. R., \& Clifford, C. W. G. (2013). Gaze categorization under uncertainty: Psychophysics and modeling. Journal of Vision, 13(5), 18-18. https://doi.org/10.1167/13.5.18 
Martinez-Conde, S., Macknik, S. L., Troncoso, X. G., \& Hubel, D. H. (2009). Microsaccades: A neurophysiological analysis. Trends in Neurosciences, 32(9), 463-475. https://doi.org/10.1016/j.tins.2009.05.006

McKee, D., Christie, J., \& Klein, R. (2007). On the uniqueness of attentional capture by uninformative gaze cues: Facilitation interacts with the Simon effect and is rarely followed by IOR. Canadian Journal of Experimental Psychology/Revue Canadienne de Psychologie Expérimentale, 61(4), 293-303. https://doi.org/10.1037/cjep2007029

Meyberg, S., Sinn, P., Engbert, R., \& Sommer, W. (2017). Revising the link between microsaccades and the spatial cueing of voluntary attention. Vision Research, 133, 4760. https://doi.org/10.1016/j.visres.2017.01.001

Meyberg, S., Sommer, W., \& Dimigen, O. (2017). How microsaccades relate to lateralized ERP components of spatial attention: A co-registration study. Neuropsychologia, 99, 64-80. https://doi.org/10.1016/j.neuropsychologia.2017.02.023

Mulckhuyse, M., \& Theeuwes, J. (2010). Unconscious attentional orienting to exogenous cues: A review of the literature. Acta Psychologica, 134(3), 299-309. https://doi.org/10.1016/j.actpsy.2010.03.002

Müller, H. J., \& Rabbitt, P. M. (1989). Reflexive and voluntary orienting of visual attention: Time course of activation and resistance to interruption. Journal of Experimental Psychology. Human Perception and Performance, 15(2), 315-330. https://doi.org/10.1037//00961523.15.2.315

Nakayama, K., \& Mackeben, M. (1989). Sustained and transient components of focal visual attention. Vision Research, 29(11), 1631-1647. 
Otsuka, Y. (2014). Dual-route model of the effect of head orientation on perceived gaze direction. Journal of Experimental Psychology: Human Perception and Performance; US: American Psychological Association. https://doi.org/10.1037/a0036151

Palanica, A., \& Itier, R. J. (2015). Eye gaze and head orientation modulate the inhibition of return for faces. Attention, Perception, \& Psychophysics, 77(8), 2589-2600. https://doi.org/10.3758/s13414-015-0961-y

Palanica, A., \& Itier, R. J. (2017). Asymmetry in Gaze Direction Discrimination Between the Upper and Lower Visual Fields. Perception, 46(8), 941-955. https://doi.org/10.1177/0301006616686989

Pastukhov, A., \& Braun, J. (2010). Rare but precious: Microsaccades are highly informative about attentional allocation. Vision Research, 50(12), 1173-1184. https://doi.org/10.1016/j.visres.2010.04.007

Perrett, D. I., Harries, M. H., Bevan, R., Thomas, S., Benson, P. J., Mistlin, A. J., Chitty, A. J., Hietanen, J. K., \& Ortega, J. E. (1989). Frameworks of analysis for the neural representation of animate objects and actions. The Journal of Experimental Biology, 146, 87-113.

Perrett, D. I., Hietanen, J. K., Oram, M. W., Benson, P. J., \& Rolls, E. T. (1992). Organization and Functions of Cells Responsive to Faces in the Temporal Cortex [and Discussion]. Philosophical Transactions: Biological Sciences, 335(1273), 23-30. JSTOR.

Perrett, D. I., Smith, P. A., Potter, D. D., Mistlin, A. J., Head, A. S., Milner, A. D., \& Jeeves, M. A. (1985). Visual cells in the temporal cortex sensitive to face view and gaze direction. 
Proceedings of the Royal Society of London. Series B, Biological Sciences, 223(1232), 293-317. https://doi.org/10.1098/rspb.1985.0003

Pestilli, F., \& Carrasco, M. (2005). Attention enhances contrast sensitivity at cued and impairs it at uncued locations. Vision Research, 45(14), 1867-1875.

Poletti, M., Rucci, M., \& Carrasco, M. (2017). Selective attention within the foveola. Nature Neuroscience, 20(10), 1413-1417. https://doi.org/10.1038/nn.4622

Posner, \& Cohen. (1984). Components of Visual Orienting. https://www.researchgate.net/profile/Yoav_Cohen/publication/203918232_Componen ts_of_visual_orienting/links/0c960516ab4213e497000000/Components-of-visualorienting.pdf

Posner, M. I. (1980). Orienting of attention. The Quarterly Journal of Experimental Psychology, 32(1), 3-25.

Ristic, J., Wright, A., \& Kingstone, A. (2007). Attentional control and reflexive orienting to gaze and arrow cues. Psychonomic Bulletin \& Review, 14(5), 964-969. https://doi.org/10.3758/BF03194129

Rolfs, M., Engbert, R., \& Kliegl, R. (2004). Microsaccade Orientation Supports Attentional Enhancement Opposite a Peripheral Cue: Commentary on Tse, Sheinberg, and Logothetis (2003). Psychological Science, 15(10), 705-707. https://doi.org/10.1111/j.0956-7976.2004.00744.x

Shi, J., Weng, X., He, S., \& Jiang, Y. (2010). Biological motion cues trigger reflexive attentional orienting. Cognition, 117(3), 348-354. https://doi.org/10.1016/j.cognition.2010.09.001 
Theeuwes, J. (1991). Exogenous and endogenous control of attention: The effect of visual onsets and offsets. Perception \& Psychophysics, 49(1), 83-90.

Võ, M. L.-H., Boettcher, S. E., \& Draschkow, D. (2019). Reading scenes: How scene grammar guides attention and aids perception in real-world environments. Current Opinion in Psychology, 29, 205-210. https://doi.org/10.1016/j.copsyc.2019.03.009

Wang, L., Yang, X., Shi, J., \& Jiang, Y. (2014). The feet have it: Local biological motion cues trigger reflexive attentional orienting in the brain. Neurolmage, 84, 217-224. https://doi.org/10.1016/j.neuroimage.2013.08.041

Yokoyama, T., \& Takeda, Y. (2019). Gaze Cuing Effects in Peripheral Vision. Frontiers in Psychology, 10, 708. https://doi.org/10.3389/fpsyg.2019.00708

Yuval-Greenberg, S., Merriam, E. P., \& Heeger, D. J. (2014). Spontaneous Microsaccades Reflect Shifts in Covert Attention. Journal of Neuroscience, 34(41), 13693-13700. https://doi.org/10.1523/JNEUROSCI.0582-14.2014

Zhao, J., Wang, L., Wang, Y., Weng, X., Li, S., \& Jiang, Y. (2014). Developmental tuning of reflexive attentional effect to biological motion cues. Scientific Reports, 4(1), 5558. https://doi.org/10.1038/srep05558 


\section{Appendix}

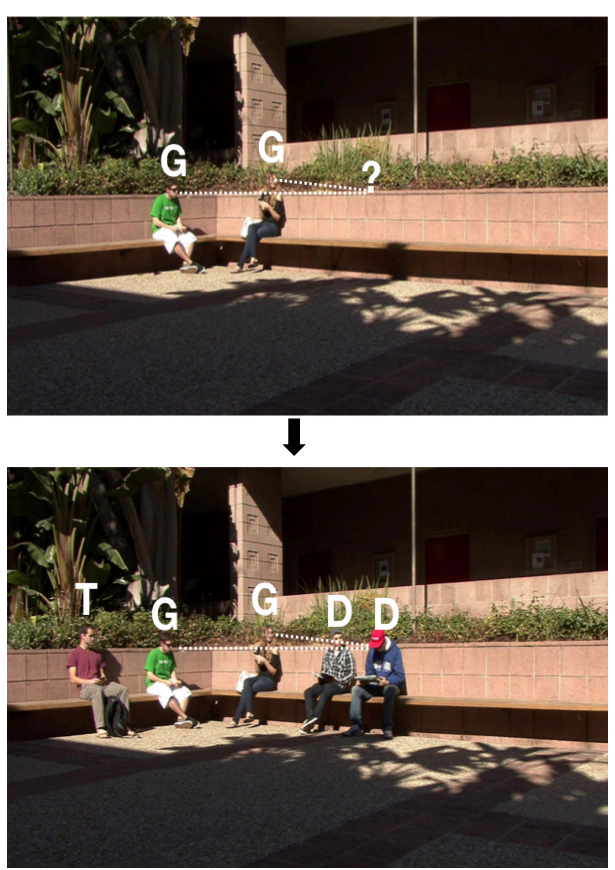

(a) Intact

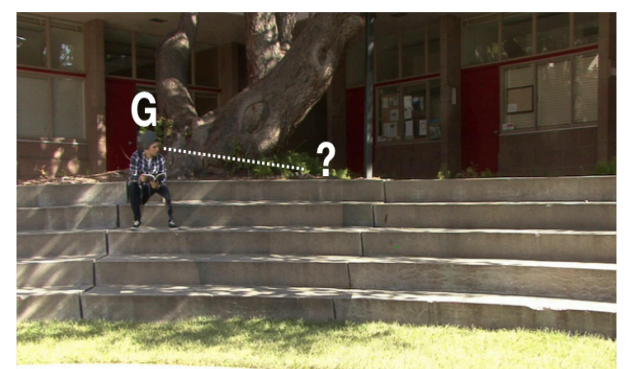

\section{$\downarrow$}

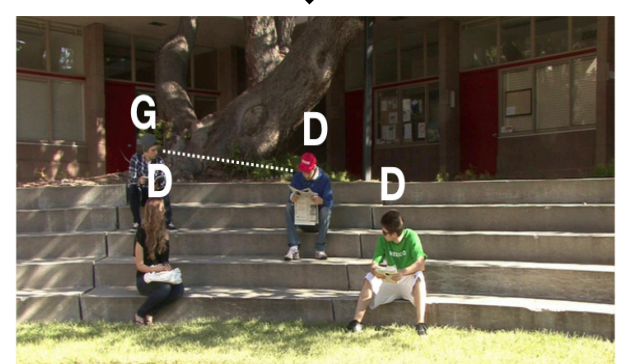

(d) Intact
Target Present - Invalid Gaze Cue

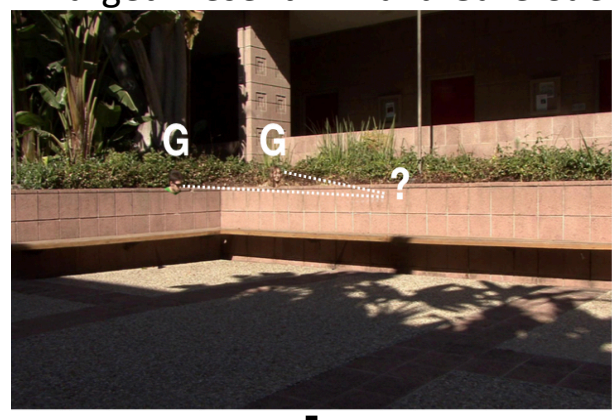

$\downarrow$

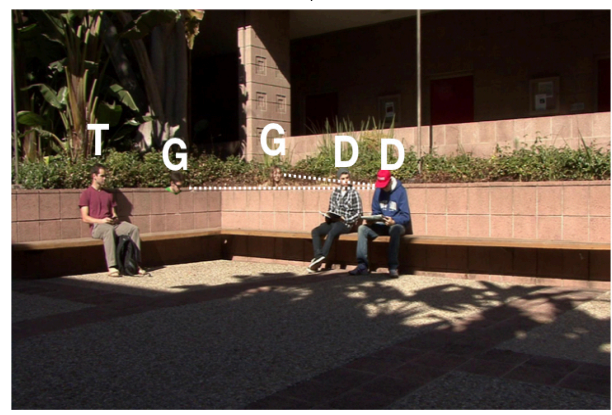

(b) floating heads

Target Absent
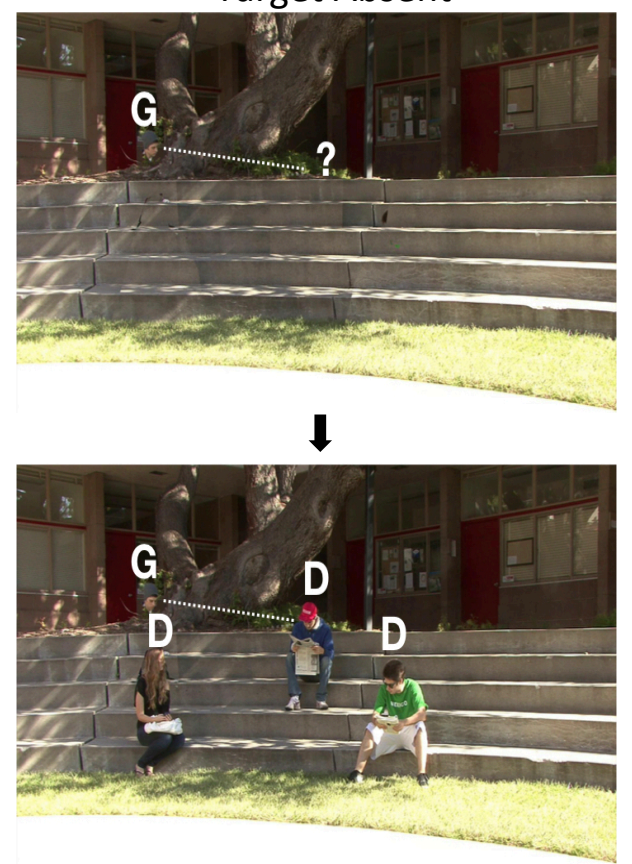

(e) floating heads

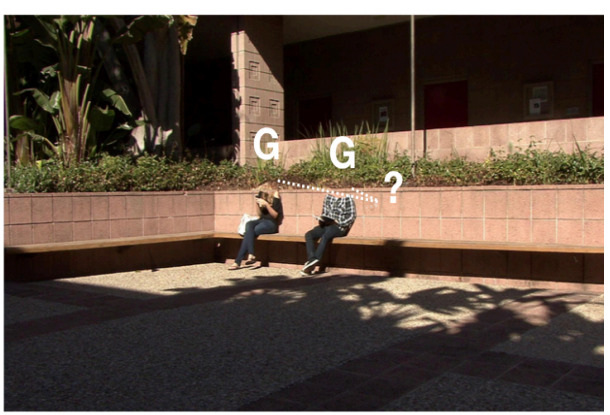

$\downarrow$

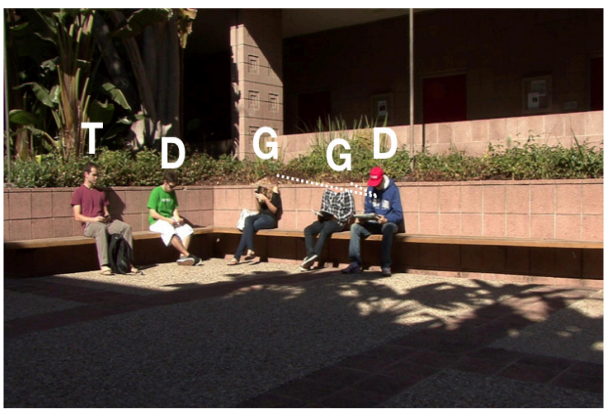

(c) headless bodies
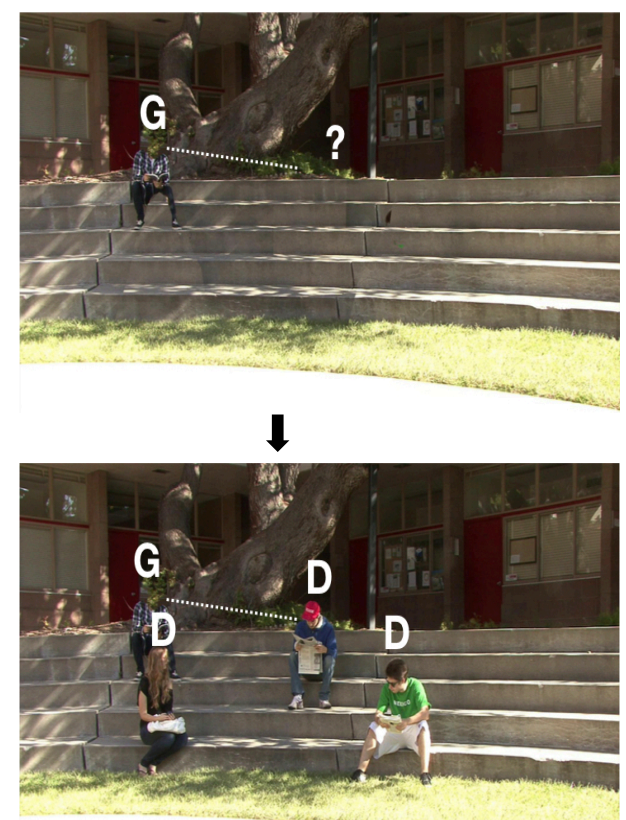

(f) headless bodies

A1. Example frames from three conditions. Gaze-orienting people (G), Target (T), Distractor (D).

(a)-(c). Target present with an invalid cue: Gaze-orienting people (G) gaze at the location with the target person (T), and two distractors (D) appeared after a $300 \mathrm{~ms}$ or $500 \mathrm{~ms}$ delay. In this case, a distractor (D) instead of the target (T) appeared at the gazed-at location, so the gaze cue was invalid. 
(d)-(f). Target absent: Gaze-orienting people (G) gazed at the location with three distractors (D) showing up after a $300 \mathrm{~ms}$ or $500 \mathrm{~ms}$ delay.

All the letter annotations and dashed lines that indicate the gaze directions were not included in the actual experiment videos.

intact

Cue Validity $\square$ invalid $\square$ valid

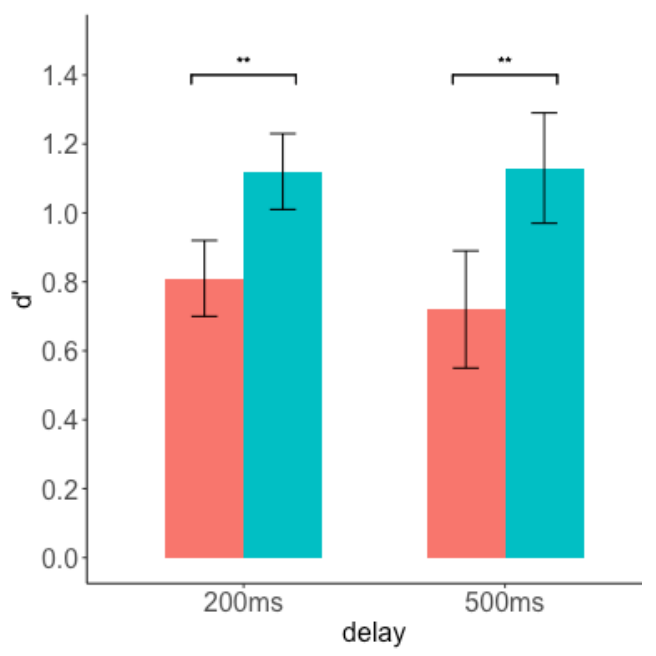

(a) floating heads

Cue Validity $\square$ invalid $\square$ valid

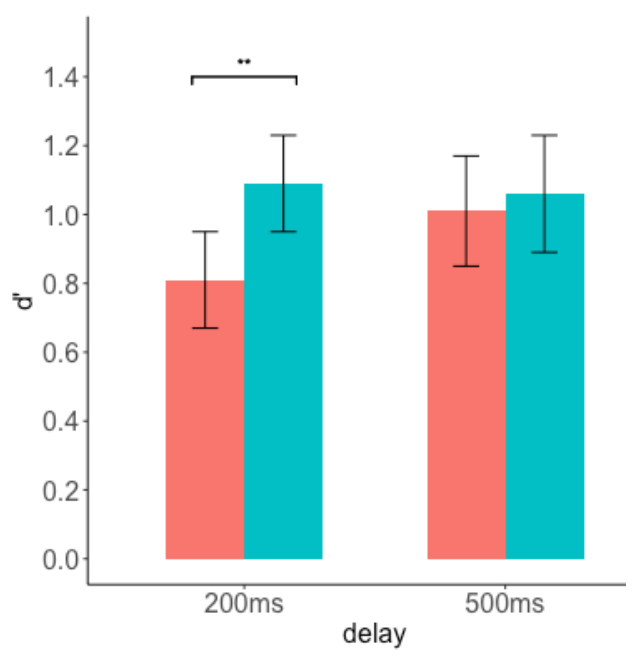

(b) headless bodies Cue Validity $\square$ invalid $\square$ valid

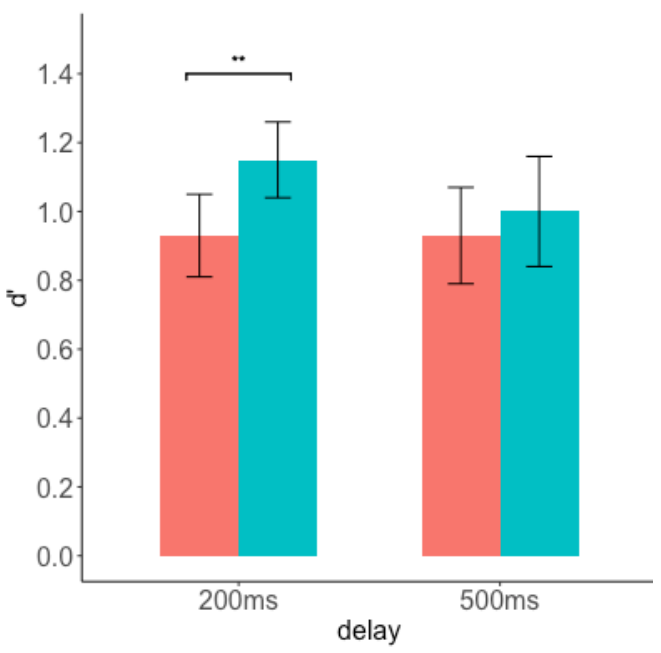

(c)

A2. d' (sensitivity) in all three conditions. 


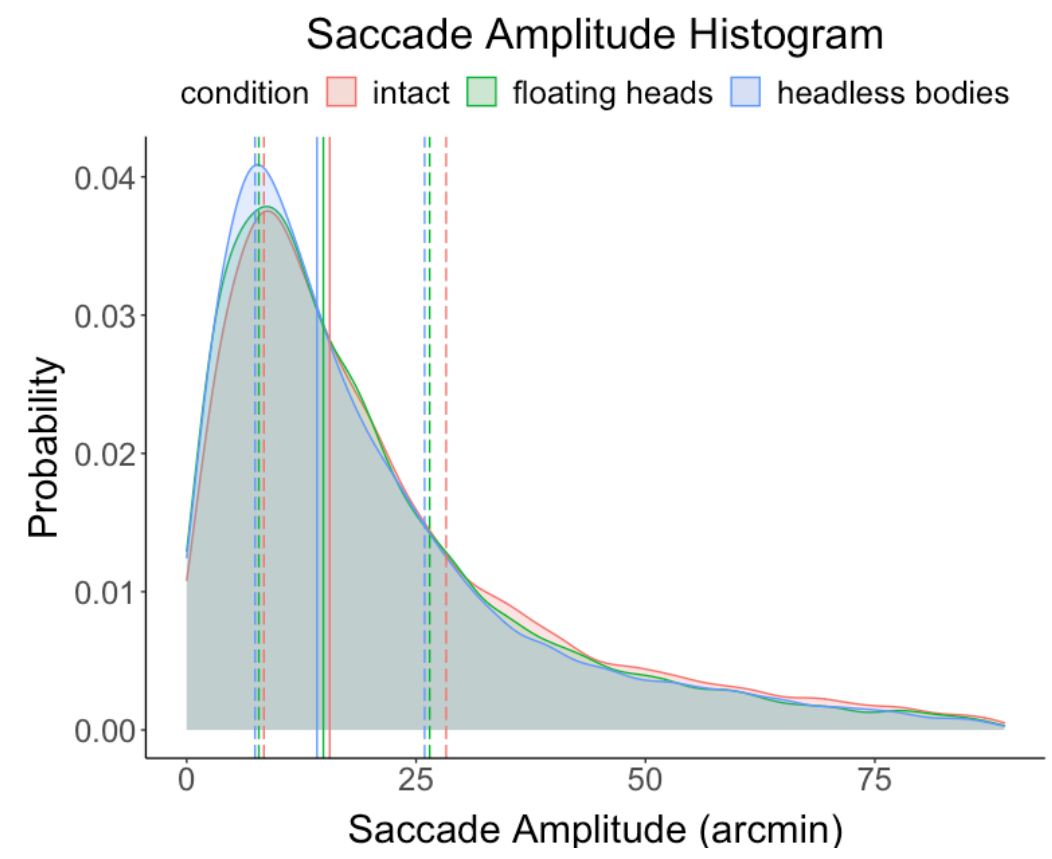

A3. Microsaccade amplitude histogram in all three conditions. $79.64 \%$ of all microsaccades were less than 30 arcmins $\left(0.5^{\circ}\right)$. Vertical lines are medians, and dashed lines are $25 \%-75 \%$ interquartile for each condition. 
Floating Heads $-\mathbf{2 0 0}$ ms

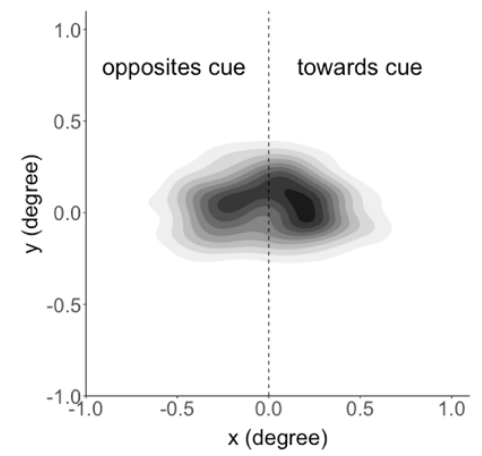

(a) Before Presentation

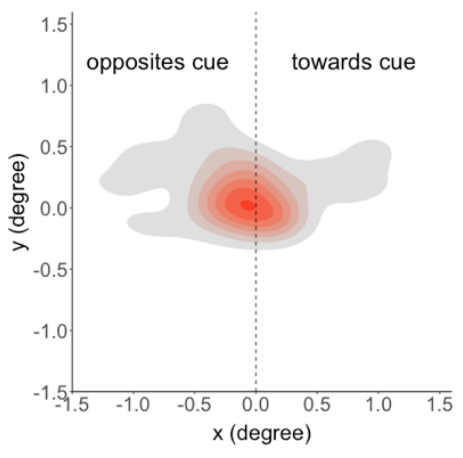

Invalid

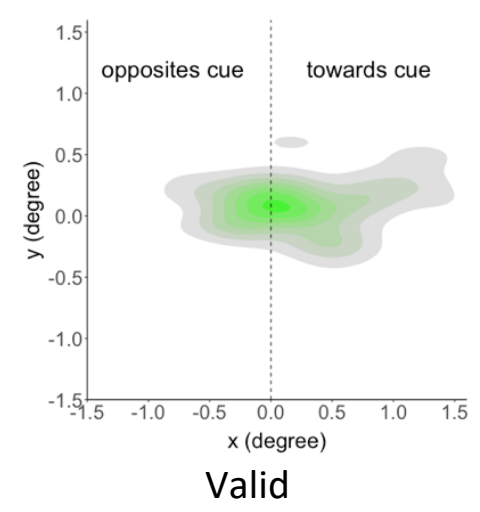

(c) $400-800 \mathrm{~ms}$ after Target/Distractor(s) Presentation

- all - valid cue - invalid cue

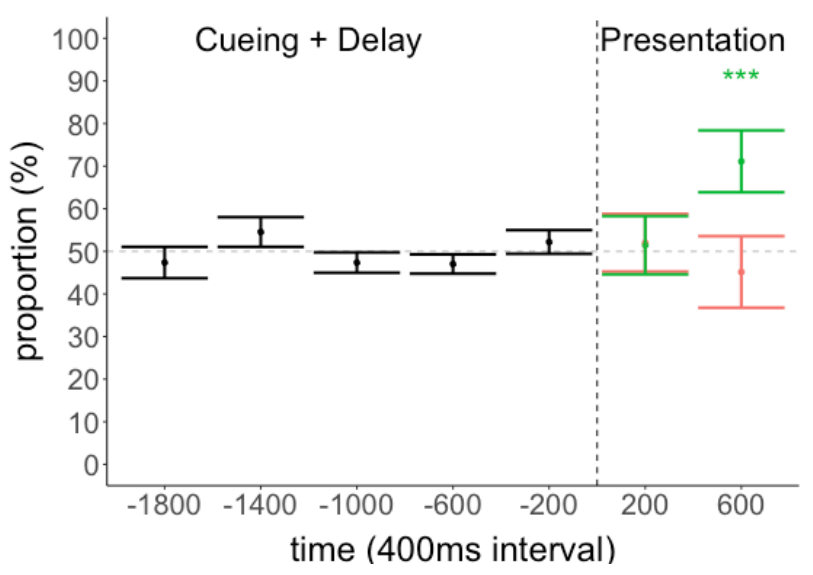

(e)

\section{Floating Heads $\mathbf{- 5 0 0} \mathrm{ms}$}

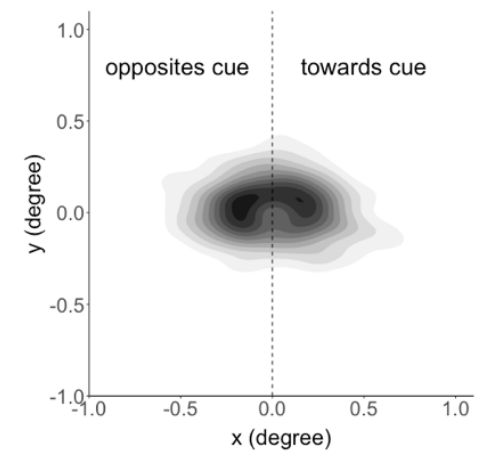

(b) Before Presentation
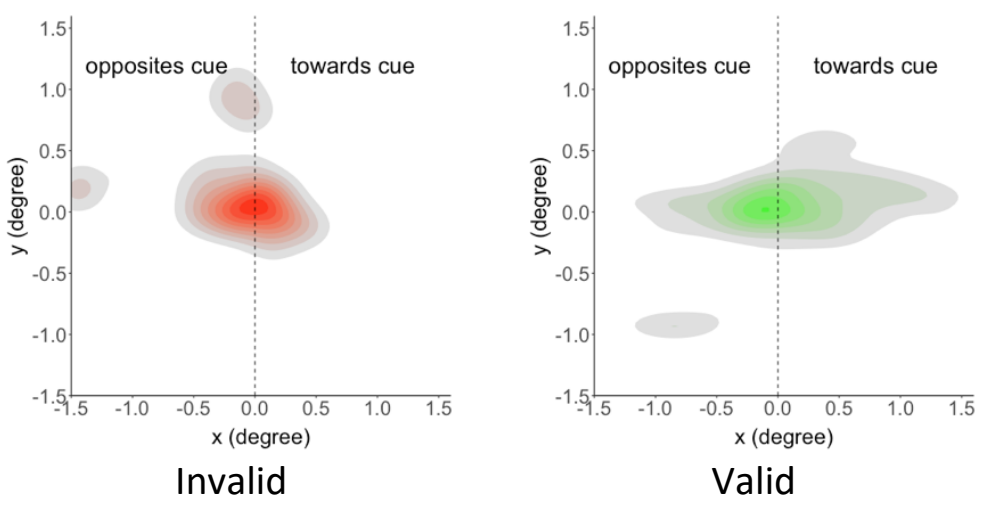

(d) $400-800 \mathrm{~ms}$ after Target/Distractor(s) Presentation - all - valid cue - invalid cue

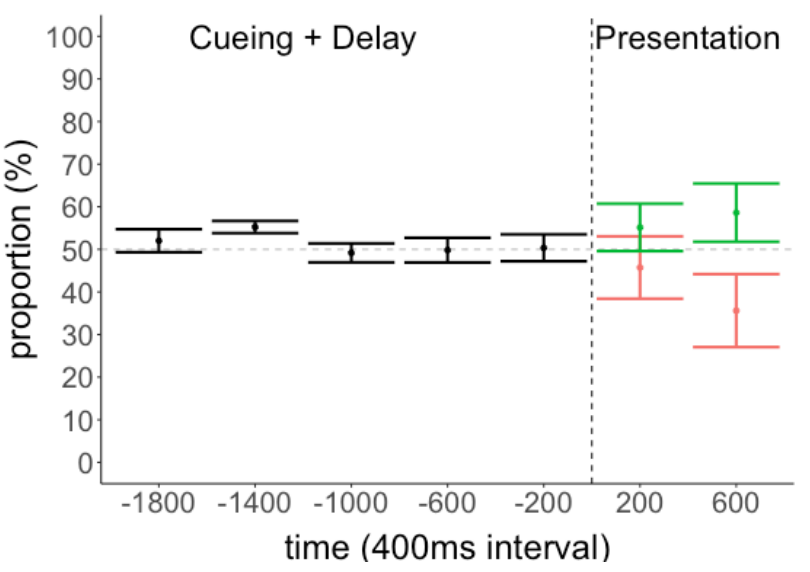

(f)

A4. Condition floating heads with $200 \mathrm{~ms}$ and $500 \mathrm{~ms}$ SOA (a)-(b) Heatmaps of microsaccades for the period before the target/distractors presentation with black and white colors (a darker color corresponds to higher density). (c)-(d) Heatmaps of microsaccades during 400-800 ms after target/distractor presentation (red color for invalid trials, green color for valid trials). (e)-(f) Proportion of microsaccades that moved toward the cued direction as a function of time for intact condition $200 \mathrm{~ms}$ and $500 \mathrm{~ms}$ delay over time. The $\mathrm{x}$-axis indicates the temporal midpoint of the time window (200 represents the time window $0-400 \mathrm{~ms}$, etc.). Times are lined up with respect to target and distractor presentation. The $y$-axis is the mean proportion of microsaccades that moved toward the cue direction. 

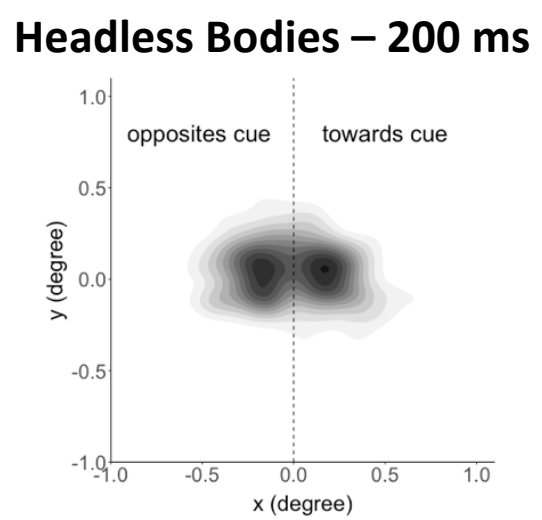

(e) Before Presentation

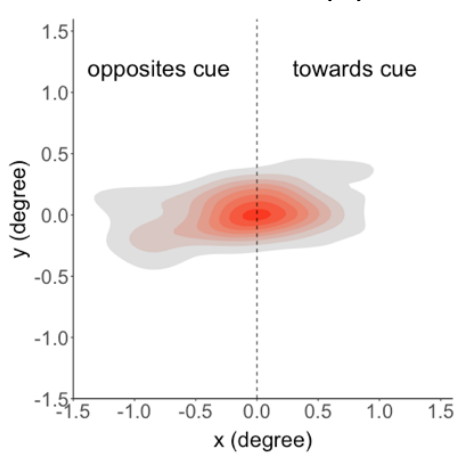

Invalid

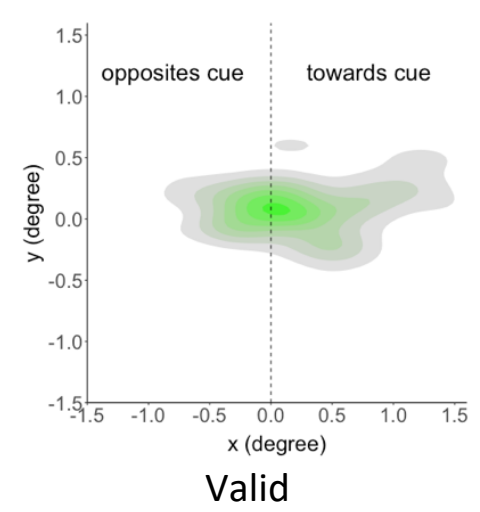

(g) 400-800ms after Target/Distractor(s) Presentation

- all - valid cue - invalid cue

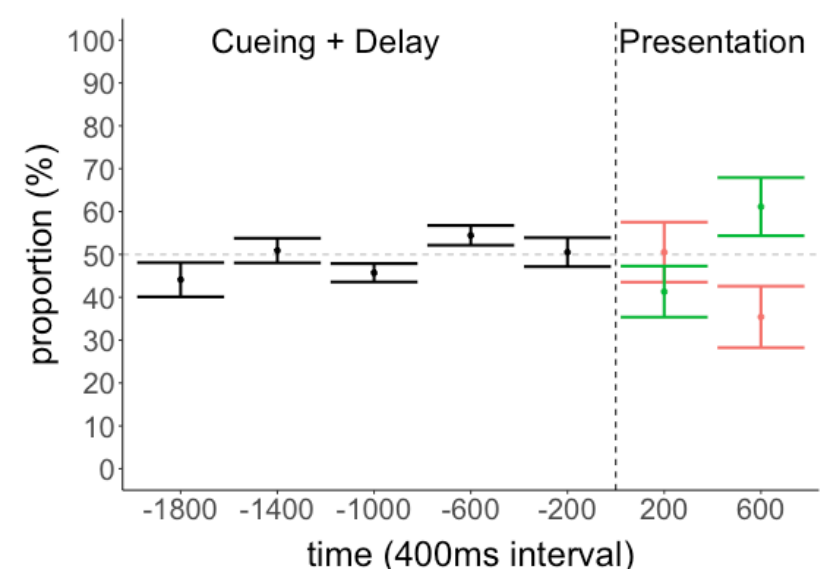

(e)

\section{Floating Heads $\mathbf{- 5 0 0} \mathrm{ms}$}

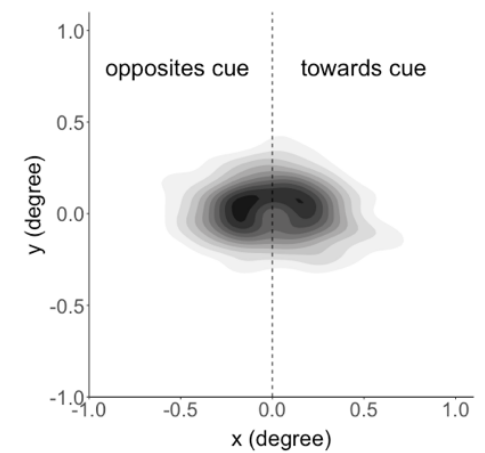

(f) Before Presentation

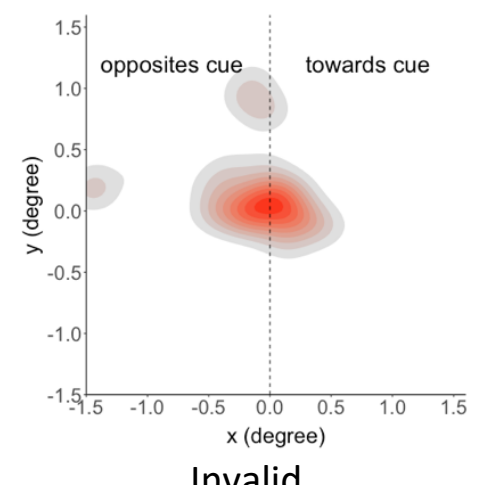

Invalid

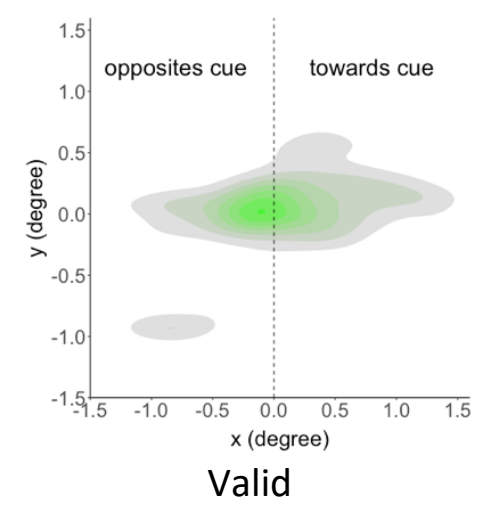

(h) 400-800ms after Target/Distractor(s) Presentation - all - valid cue - invalid cue

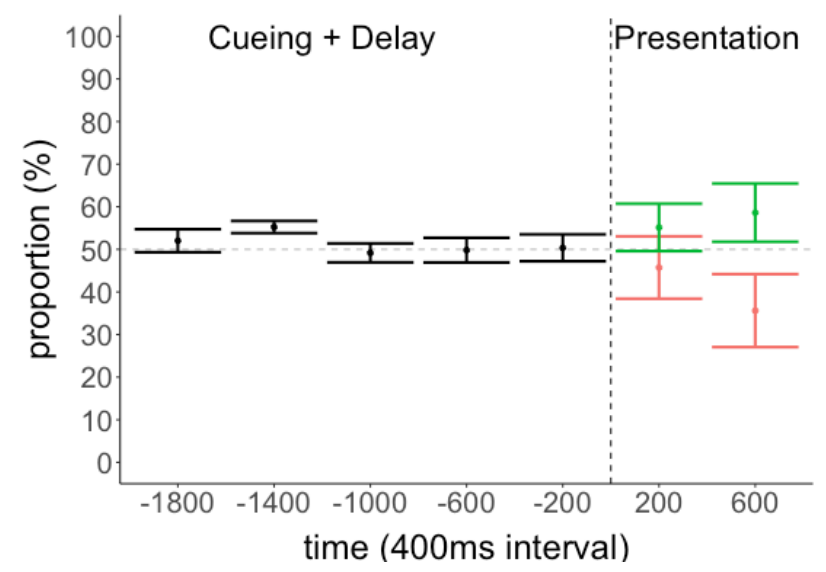

(f)

A5. Condition headless bodies with $200 \mathrm{~ms}$ and 500ms SOA (a)-(b) Heatmaps of microsaccades for the period before the target/distractors presentation with black and white colors (a darker color corresponds to higher density). (c)-(d) Heatmaps of microsaccades during 400-800 ms after target/distractor presentation (red color for invalid trials, green color for valid trials). (e)-(f) Proportion of microsaccades that moved toward the cued direction as a function of time for intact condition $200 \mathrm{~ms}$ and $500 \mathrm{~ms}$ delay over time. The $\mathrm{x}$-axis indicates the temporal midpoint of the time window (200 represents the time window $0-400 \mathrm{~ms}$, etc.). Times are lined up with respect to target and distractor presentation. The $y$-axis is the mean proportion of microsaccades that moved toward the cue direction. 


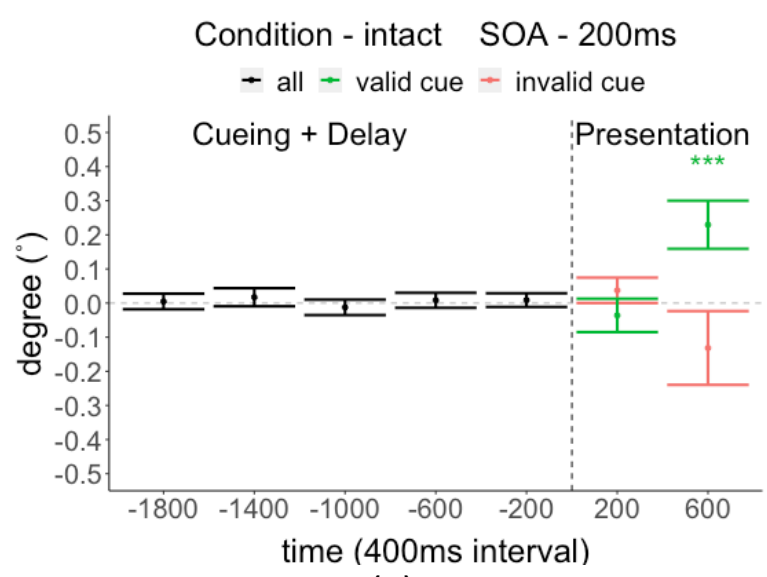

(a)

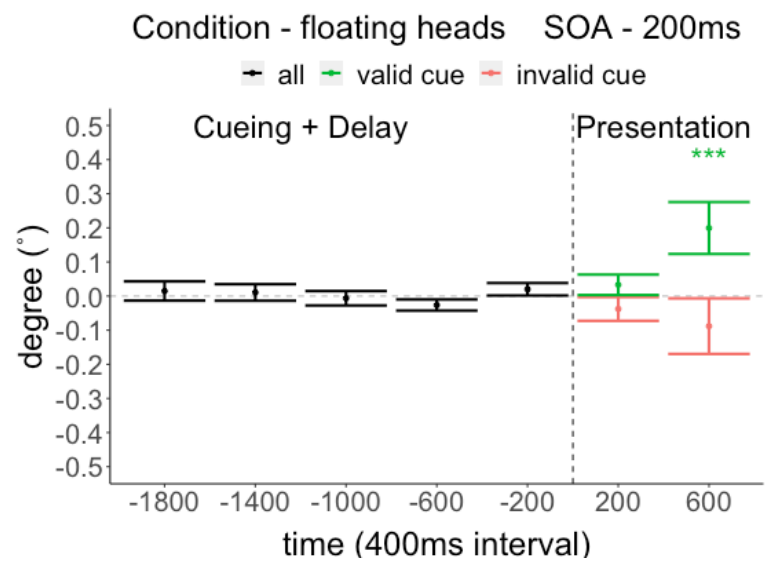

(c)

Condition - headless bodies SOA - 200ms

- all - valid cue - invalid cue

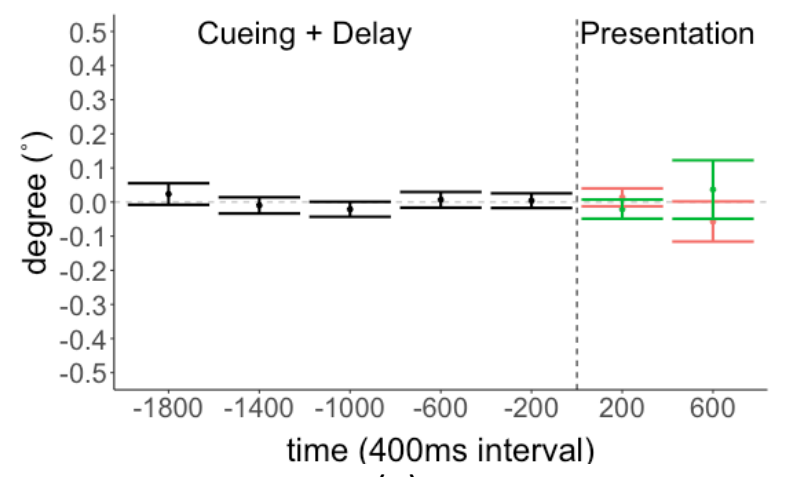

(e)

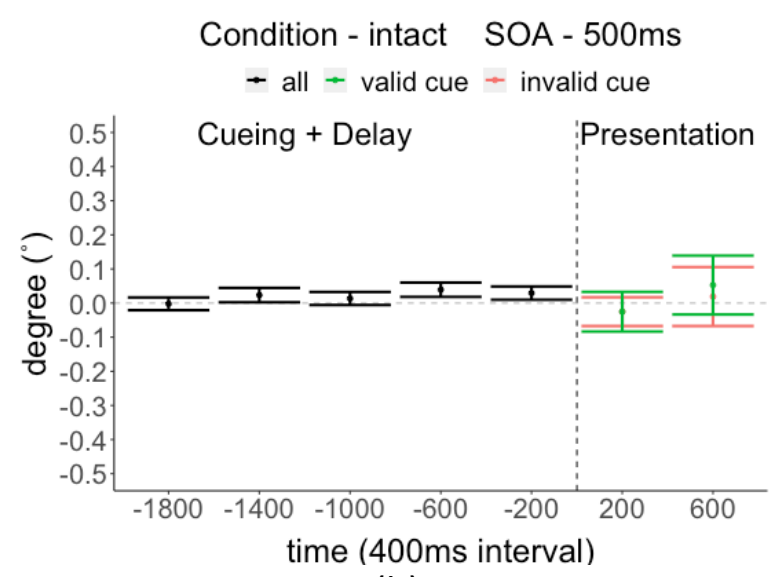

(b)

Condition - floating heads SOA - 500ms

- all - valid cue - invalid cue

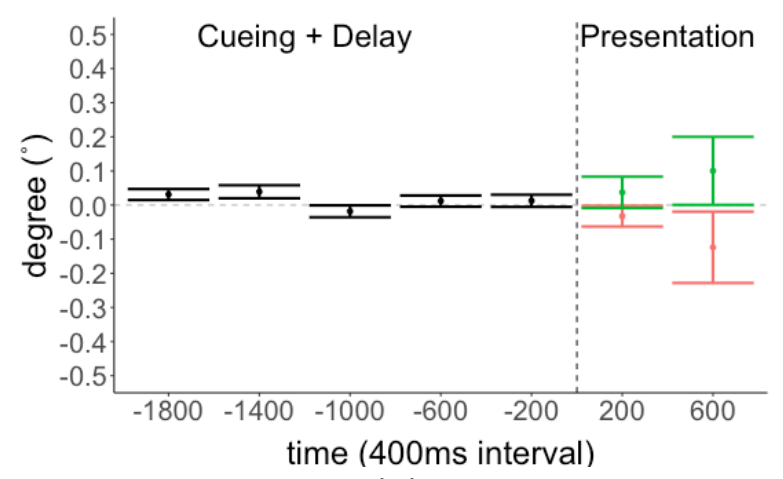

(d)

Condition - headless bodies SOA - 500ms

- all - valid cue - invalid cue

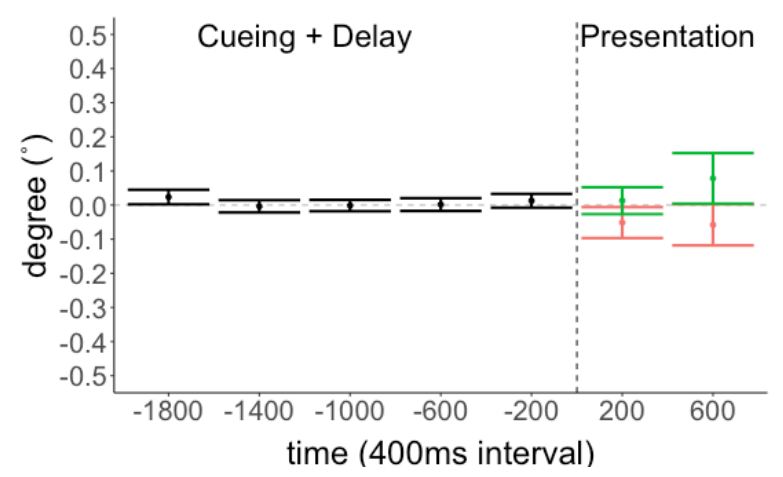

(f)

A6. Microsaccades degrees toward the cued direction (left/right side of forced fixation) as a function of time for the intact condition $200 \mathrm{~ms}$ (a) and $500 \mathrm{~ms}(\mathrm{~b})$ the floating heads condition(c)-(d), and the headless bodies condition (e)-(f). The x-axis

indicates the temporal midpoint of the time window (200 represents time window $0-400 \mathrm{~ms}$, etc.). The $y$-axis is the mean deflection in degrees in visual angle toward the cue direction (positive values represent that the microsaccades are biased toward the cue direction). Black bars represent microsaccades before target/distractors presentation averaged across both valid and invalid trials. Green and red bars represent microsaccades after target/distractors presentation for valid and invalid trials, respectively. 


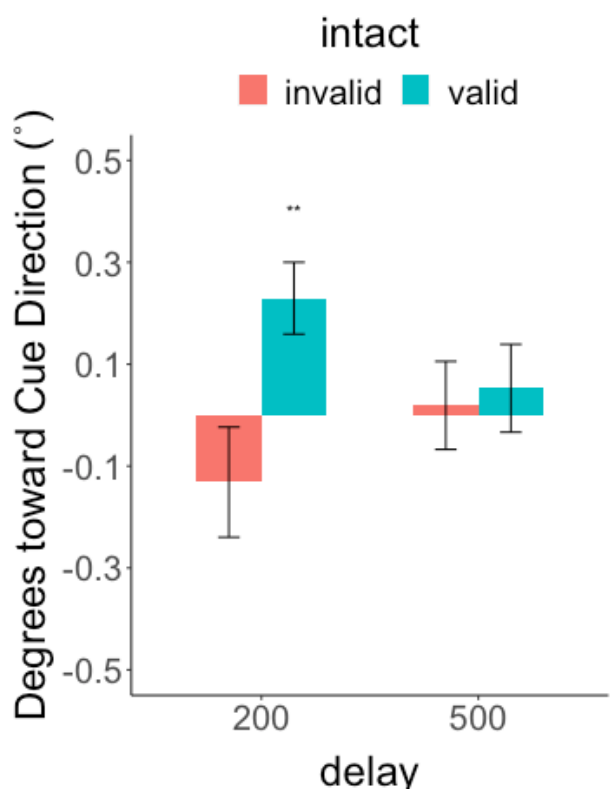

(a)

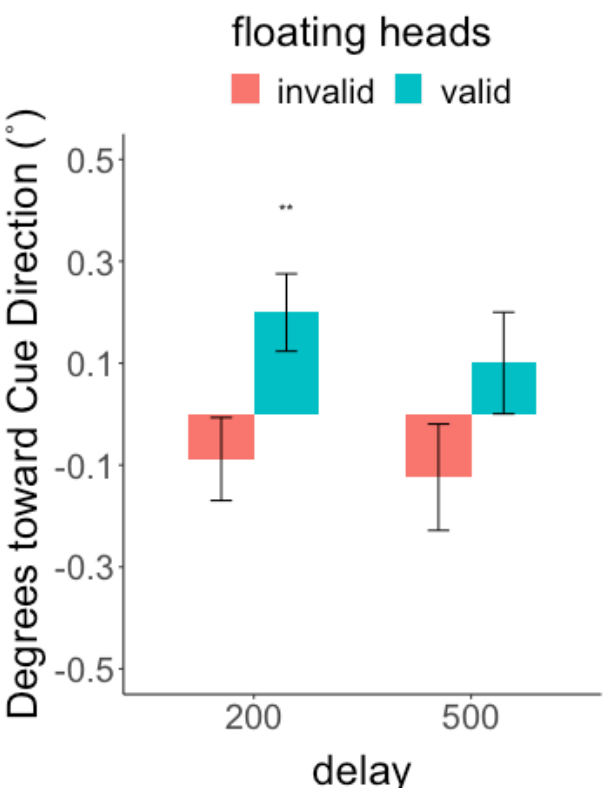

(b)

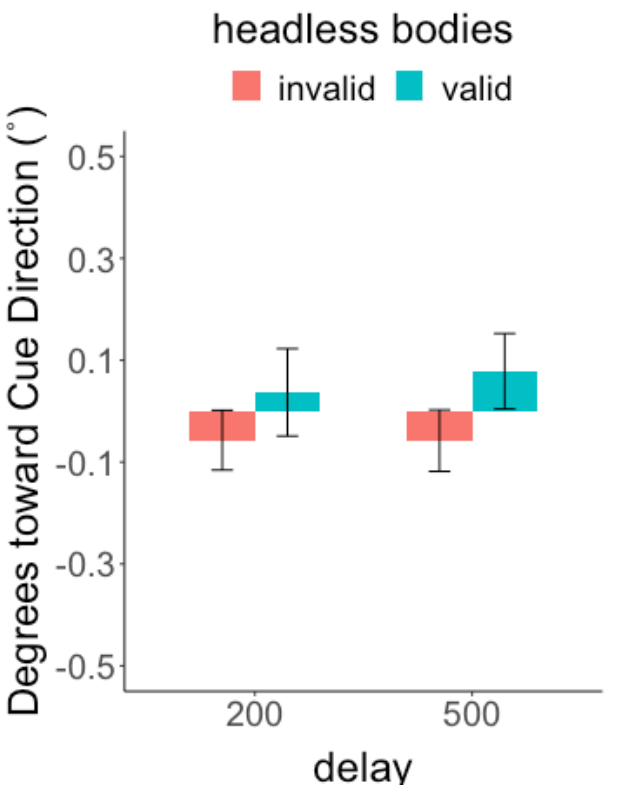

(c)

A7. Microsaccades degrees toward the cued direction summarized within $400 \mathrm{~ms}-800 \mathrm{~ms}$ after target/distractors presentation onset for intact condition (a), floating heads condition (b), and headless bodies condition (c). 